\title{
The Drosophila SK Channel (dSK) Contributes to Photoreceptor Performance by Mediating Sensitivity Control at the First Visual Network
}

\author{
Ahmad N. Abou Tayoun, ${ }^{1 *}$ Xiaofeng Li, ${ }^{2,3 *}$ Brian Chu, ${ }^{4}$ Roger C. Hardie, ${ }^{4}$ Mikko Juusola, ${ }^{2,3}$ and Patrick J. Dolph ${ }^{1}$ \\ ${ }^{1}$ Department of Biology, Dartmouth College, Hanover, New Hampshire 03755, ${ }^{2}$ Department of Biomedical Science, University of Sheffield, Sheffield S10 \\ 2TN, United Kingdom, ${ }^{3}$ State Key Laboratory of Cognitive Neuroscience and Learning, Beijing Normal University, Beijing 100875, China, and ${ }^{4}$ Department \\ of Physiology, Development, and Neuroscience, University of Cambridge, Cambridge CB2 3DY, United Kingdom
}

The contribution of the SK (small-conductance calcium-activated potassium) channel to neuronal functions in complex circuits underlying sensory processing and behavior is largely unknown in the absence of suitable animal models. Here, we generated a Drosophila line that lacks the single highly conserved $S K$ gene in its genome $(d S K)$. In R1-R6 photoreceptors, $d S K$ encodes a slow $\mathrm{Ca}^{2+}$-activated $\mathrm{K}^{+}$ current similar to its mammalian counterparts. Compared with wild-type, $d S K^{-}$photoreceptors and interneurons showed accelerated oscillatory responses and adaptation. These enhanced kinetics were accompanied with more depolarized $d S K^{-}$photoreceptors axons, assigning a role for dSK in network gain control during light-to-dark transitions. However, compensatory network adaptation, through increasing activity between synaptic neighbors, overcame many detriments of missing dSK current enabling $d S K^{-}$photoreceptors to maintain normal information transfer rates to naturalistic stimuli. While demonstrating important functional roles for dSK channel in the visual circuitry, these results also clarify how homeostatically balanced network functions can compensate missing or faulty ion channels.

\section{Introduction}

Small-conductance calcium-activated potassium (SK) channels play a fundamental role in excitable cells, linking changes in intracellular $\mathrm{Ca}^{2+}$ to membrane potential (Stocker, 2004). SK channels are voltage-independent and upon indirect activation by $\mathrm{Ca}^{2+}$ through a constitutively bound calmodulin (Xia et al., 1998), they cause membrane hyperpolarization, thus inhibiting cell firing and shaping frequency of repetitive action potentials (Wolfart et al., 2001; Hallworth et al., 2003). At the synapse, SK channels couple to calcium sources and negatively regulate synaptic transmission and plasticity underlying learning and memory (Faber et al., 2005; Ngo-Anh et al., 2005). SK channels seem also important in refining communication and connectivity in sensory systems. In the mammalian retina, SK is expressed in the retinal ganglion cells, in the horizontal and the dopaminergic amacrine cells of the inner nuclear layer, and have been implicated in activity-dependent plasticity during development

\footnotetext{
Received June 20, 2011; revised July 19, 2011; accepted July 25, 2011.

Author contributions: R.C.H., M.J., and P.J.D. designed research; A.N.A.T., X.L., and B.C. performed research; A.N.A.T., X.L., B.C., R.C.H., M.J., and P.J.D. analyzed data; A.N.A.T., X.L., M.J., and P.J.D. wrote the paper.

This work was supported in part by a grant from the National Institutes of Health (P.J.D.) and by Biotechnology and Biological Sciences Research Council Grants BBF0120711, BBD00119001, and BBH0138491 (M.J.). We thank Ann M. Lavanway for her assistance with confocal microscopy.

*A.N.A.T. and X.L. contributed equally to this work.

Correspondence should be addressed to either of the following: Patrick J. Dolph, Department of Biology, Dartmouth College, Hanover, NH 03755, E-mail: Patrick.Dolph@dartmouth.edu; or Mikko Juusola, Department of Biomedical Science, University of Sheffield, Sheffield S10 2TN, UK, E-mail: M.Juusola@Sheffield.ac.uk.

DOI:10.1523/JNEUROSCI.3134-11.2011

Copyright $\odot 2011$ the authors $\quad 0270-6474 / 11 / 3113897-14 \$ 15.00 / 0$
}

(Shatz, 1990; Wang et al., 1999; Klöcker et al., 2001; Clark et al., 2009).

The vertebrate retina and the insect lamina (first optic neuropil) share the design principle of massively parallel processing, implemented through rich networks of interconnected microcircuits with multiple-contact synapses (Meinertzhagen and Sorra, 2001; Sanes and Zipursky, 2010). In the Drosophila lamina, histaminergic R1-R6 photoreceptors synapse (Hardie, 1987, 1989; Gengs et al., 2002) onto the primary interneurons: the large monopolar cells (LMCs: L1-L3), and the amacrine cells (ACs) (Meinertzhagen and O'Neil, 1991; Uusitalo et al., 1995a). In turn, L2 and AC feedback onto photoreceptor terminals via excitatory inputs (Sinakevitch and Strausfeld, 2004; Kolodziejczyk et al., 2008). Upon light stimulation, photoreceptors depolarize while LMCs/ACs hyperpolarize (Shaw, 1984; Zheng et al., 2006), which thereby reduces excitatory feedback onto the photoreceptors. Accordingly, voltage responses in the photoreceptor-LMC-photoreceptor network appear shaped together and adapt together (network adaptation), presumably to best encode and route light information over multiple pathways (Nikolaev et al., 2009; Zheng et al., 2009). Nonetheless, this simplified view of processing excludes the less studied contribution of glia and interneurons: C2-C3 fibers, L4-L5, T1 and Tan cells.

Three mammalian genes encode for similar SK1-3 subunits with overlapping expression patterns (Köhler et al., 1996). Despite the available pharmacological studies, there have been no suitable animal models to explore how SK channels affect complex network activity that underlies sensory integration and be- 


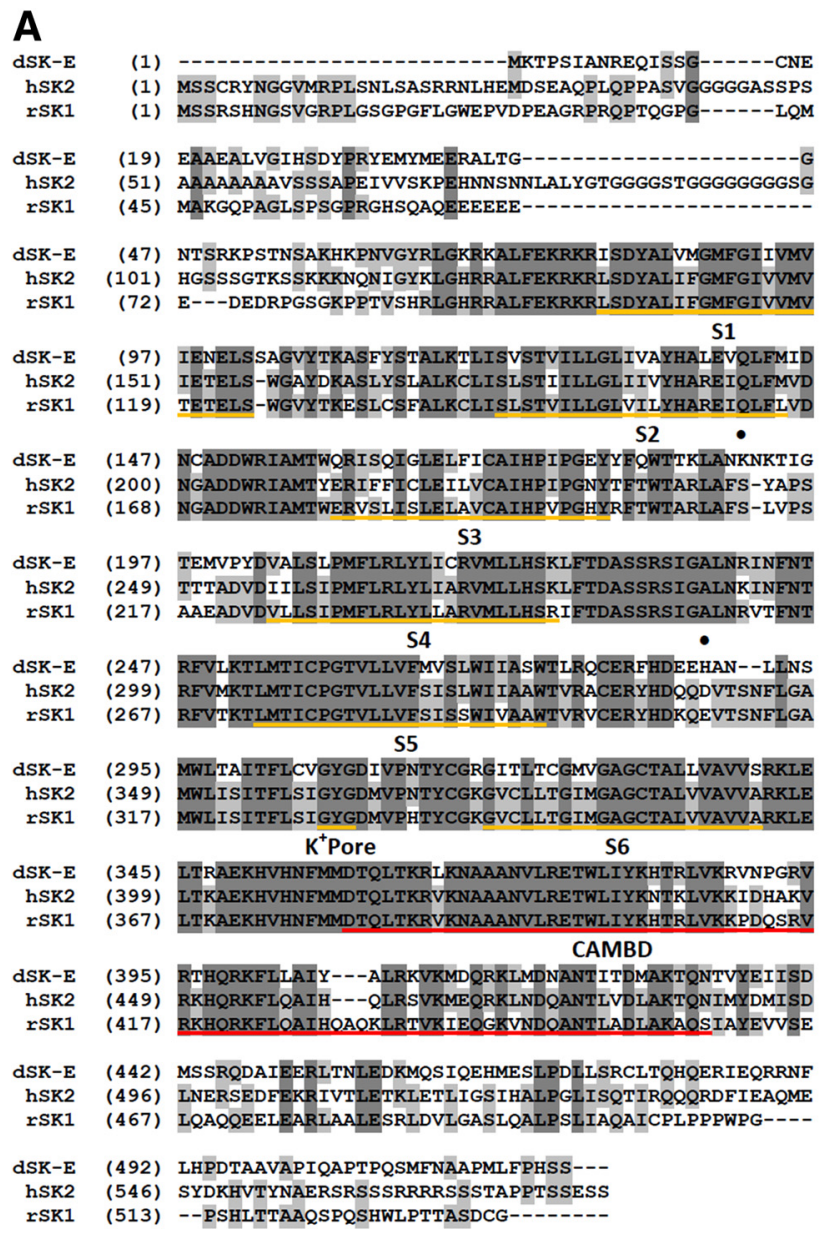

B
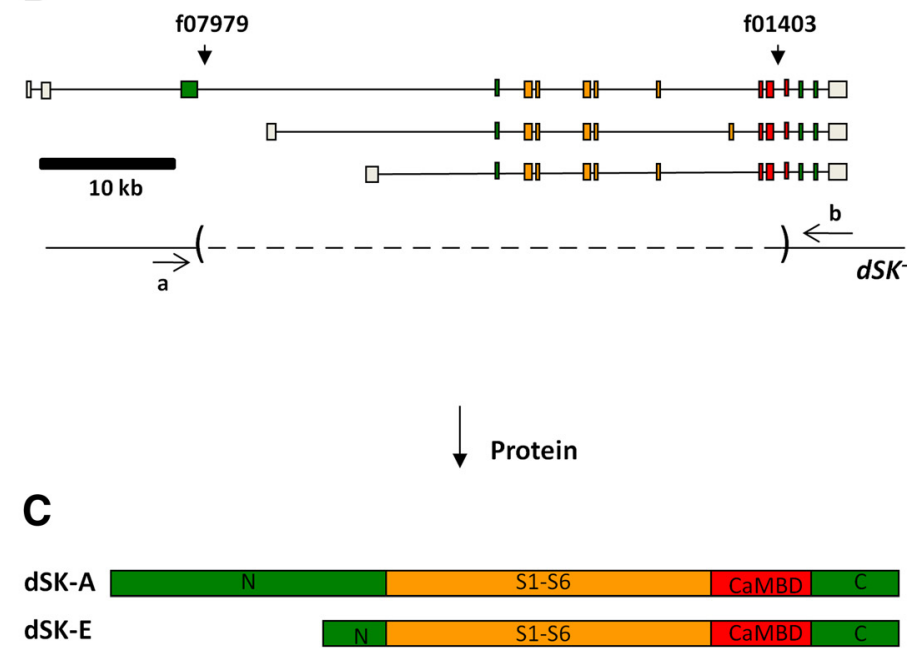

Figure 1. Structure and targeted mutation in dSK gene. $A$, Putative amino acid sequence of dSK (E isoform) and its alignment with the rat SK1 and human SK2. The underlined regions indicate the six transmembrane domains $(\mathrm{S1} 1 \mathrm{S6})$, the $\mathrm{K}^{+}$pore, and the CAMBD. Light and dark shaded areas represent conservative and identical residues, respectively. Closed circles indicate amino acids implicated in apamin sensitivity. $\boldsymbol{B}$, Organization of $d S K$ transcripts. Shown below transcripts is structure of the $d S K^{-}$allele, generated by FLP-FRT-mediated recombination using PiggyBac insertions f07979 and f01403. Dashed lines indicate deleted regions. C, Predicted dSK protein isoforms E and A. D, PCR products from whole fly genomic DNA, amplified using primers a and b indicated in $\boldsymbol{B}$. $P C R$ product size was consistent with the presence of a deletion and was further confirmed by DNA sequencing.

havior. The presence of a single SK gene in the Drosophila genome provides an opportunity to investigate the function of this channel in a well established genetic animal model.

Here we combine fly genetics with electrophysiology to show that dSK contributes to photoreceptor performance by mediating sensitivity control at the lamina network. $d S K^{-}$flies exhibited fast, oscillatory network responses leading to fast adapting photoreceptors with a slightly broader frequency bandwidth. Furthermore, mutant photoreceptors had altered physiology: lower input resistance and higher resting potential in the dark, suggesting that they entertained larger depolarizing conductances at photoreceptor axons from extrinsic inputs. This was supported by their normal somatic light-induced currents and high dSK expression in the lamina circuits. Surprisingly, $d S K^{-}$photoreceptors had normal encoding capacity, highlighting the robustness of the network adaptation for maintaining appropriate information processing.

\section{Materials and Methods}

Generation of $\mathrm{dSK}^{-}$mutant and constructs. $S K^{f 07979}$ and $S K^{f 01403}$ piggyBac lines were obtained from the Bloomington Stock Center. Using the FRT sites present in these two lines and FLP-mediated recombination we generated a deletion in the $d S K$ gene (Fig. 1). The intended modifications were verified by PCR amplification and DNA sequencing across the deleted region. The regenerated piggyBac element was precisely excised to make $w^{-} d S K^{-}$flies. These flies were outcrossed to Canton-S to establish a $w^{+} d S K^{-}$recombinant stock. The OK371-Gal4 line was obtained from the Bloomington Stock Center.

UAS-dSK (myc-tagged) rescue construct was prepared by PCR amplification of the entire coding (short SK isoform) and $5^{\prime}$ and $3^{\prime}$ untranslated regions from the EST clone GH16664, and inserting this fragment into pUAST. We designed a specific set of primers to insert a myc-tag at the $\mathrm{C}$ terminus. To generate a dominant-negative dSK subunit (UAS-SKDNmyc), we used site-directed mutagenesis to change the $\mathrm{K}^{+}$pore residues (GYG) in the rescue construct into (AAA). The dominant-negative mutation and the myc-tag insertions were confirmed by DNA sequencing.

Production of anti-dSK antibody and Western blot analysis. We generated anti-dSK antibody against the N-terminal domain of the protein. The region encoding the full $\mathrm{N}$-terminal portion of the long dSK protein isoform (first $480 \mathrm{aa}$ ) was reconstituted using double-PCR from the EST clones GH16664 and GH01258, which contained the regions encoding the last 80 aa and the first 415 aa of the $N$ terminus, respectively. This reconstituted fragment was subcloned into the pMAL-c2x and pGEX4T.1 vectors. The MBP fusion protein was affinity purified and polyclonal antisera were generated offsite (Covance). dSK antibodies from animal bleeds were affinity purified using a SulfoLink column (Pierce) coupled to GST fusion proteins.

Western blot analysis was performed on membrane proteins as previously described (Kwon and Montell, 2006). Briefly, fly heads of either sex were homogenized in $1 \times$ PBS solution and incubated on ice for $15 \mathrm{~min}$. Following a maximum speed centrifugation for $10 \mathrm{~min}$ in a Microfuge, 
the pellet was resuspended in $1 \times$ PBS solution, containing $1 \%$ Triton $\mathrm{X}-100$ and incubated on ice for $15 \mathrm{~min}$. After centrifugation for $10 \mathrm{~min}$ at $8000 \mathrm{rpm}$, the supernatants were separated by SDS-PAGE, transferred into nitrocellulose membranes and the protein bands were detected by Western blot analysis with dSK antibodies at a 1:2500 dilution.

Protein sequence analysis. Protein sequence alignment was performed using Vector NTI software from Invitrogen. dSK membrane topology was predicted by running a Kyte and Doolittle algorithm in Gene Inspector software.

Immunohistochemistry. Adult fly heads of either sex were fixed in $4 \%$ paraformaldehyde solution in PBS for $3 \mathrm{~h}$. Heads were then washed five times for 5 min with PBS, containing $0.2 \%$ Triton X-100 (PBT). Brains were then dissected in ice-cold PBT, washed two times for $10 \mathrm{~min}$ with PBT, blocked for $1 \mathrm{~h}$ with PBT, containing 3\% normal goat serum, and incubated with primary antibody in blocking solution for $1 \mathrm{~d}$ at room temperature. Samples were washed five times for $5 \mathrm{~min}$ with PBT, incubated with secondary antibodies in blocking solution for $2 \mathrm{~h}$ at room temperature, washed three times for $5 \mathrm{~min}$, and mounted in Vectashield (Vector Labs) for confocal microscopy. Imaging was performed using a Nikon A1RSi confocal microscope and the Nikon Elements software. dSK antibodies were used at a 1:2000 dilution. nc82 and 24B10 (Developmental Studies Hybridoma Bank, University of Iowa) antibodies were used at 1:50.

Eye sections. Heads of dark-reared flies of either sex were bisected, fixed, and embedded as previously described (Chinchore et al., 2009). Eye cross sections $(1 \mu \mathrm{m})$ were cut using a Sorvall ultra microtome MT-1, stained with toluidine blue and borax, and observed using a Zeiss Axioplan2 microscope. Digital images were captured using Optronics DEI-750 camera and MetaVue (Universal Imaging) software.

Whole-cell recordings. Dissociated ommatidia were prepared from recently eclosed adult flies of either sex and transferred to a recording chamber on an inverted Nikon Diaphot microscope (Hardie et al., 2002). The control bath solution contained the following (in mM): $120 \mathrm{NaCl}, 5$ $\mathrm{KCl}, 10 \mathrm{~N}$-Tris-(hydroxymethyl)-methyl-2-aminoethanesulfonic acid (TES), $4 \mathrm{MgCl}_{2}, 1.5 \mathrm{CaCl}_{2}, 25$ proline, and 5 alanine. Osmolarity was adjusted to $\sim 283 \mathrm{mOsm}$. The standard intracellular solution used in the recording pipette was composed of the following (in $\mathrm{mm}$ ): $140 \mathrm{~K}^{+}$gluconate, 10 TES, $4 \mathrm{Mg}^{2+}$ ATP, $2 \mathrm{MgCl}_{2}, 1 \mathrm{NAD}$, and $0.4 \mathrm{Na}^{+}$GTP. For $\mathrm{Na}^{+} / \mathrm{Ca}^{2+}$ exchange study, cells were exposed to $50-100 \mu \mathrm{M}$ ouabain for $\sim 1$ min before recording to block $\mathrm{Na}^{+} / \mathrm{K}^{+}$ATPase. Extracellular $\mathrm{Na}^{+}$ concentration was varied from 120 to $0 \mathrm{~mm}$ by equimolar substitution with $\mathrm{CsCl}, \mathrm{KCl}$, or $\mathrm{LiCl}$. The intracellular solution also contained either 10 or $20 \mathrm{~mm} \mathrm{NaCl}$, and $\mathrm{Cs}^{+} / \mathrm{K}^{+}$gluconate reduced as necessary to maintain osmolarity. For some experiments, $15 \mathrm{~mm}$ TEA was included to block voltage-sensitive $\mathrm{K}^{+}$conductance. Apamin $(5 \mu \mathrm{M})$ was included to test the apamin sensitivity of SK conductance. 1-EBIO (1-ethyl-2benzimidazolinone) was dissolved in ethanol and stored at $-20^{\circ} \mathrm{C}$ as a $0.1 \mathrm{M}$ stock solution, diluted before use, and bath-applied in control solution, containing $85 \mathrm{~mm} \mathrm{NaCl}, 40 \mathrm{~mm} \mathrm{KCl}$. The final concentration of ethanol used during 1-EBIO application $(<1 \%)$ showed no significant effects. $\mathrm{pH}$ of all solutions was adjusted to 7.15. Data were recorded at $20 \pm 1{ }^{\circ} \mathrm{C}$ with Axopatch 1-D or 200 amplifiers and analyzed with pClamp 8 or 9 software (Molecular Devices). Cells were stimulated by a green-light-emitting diode with intensities calibrated in terms of effectively absorbed photons by counting quantum bumps at low intensities in wild-type flies.

In vivo intracellular recordings. Flies of either sex were immobilized in a conical fly holder with beeswax, as previously described (Juusola and Hardie, 2001; Zheng et al., 2006). To allow the recording microelectrode to enter the retina/lamina, a small hole with the size of few ommatidia was cut in the dorsal cornea and sealed with Vaseline to prevent the eye from drying. Intracellular voltage responses were recorded through sharp quartz and borosilicate microelectrodes (Sutter Instruments), having 120-200 M $\Omega$ resistance. Recordings from R1-R6 photoreceptors and LMCs were performed separately; using $3 \mathrm{M} \mathrm{KCl}$ intra-electrode solution (photoreceptors) and $3 \mathrm{~m}$ potassium acetate with $0.5 \mathrm{~mm} \mathrm{KCl}$ (LMC; to minimize reduction in the chloride battery). A blunt reference electrode was inserted into the fly head capsule close to the ocelli. The head temperature of the flies was kept at $19 \pm 1^{\circ} \mathrm{C}$ by a feedbackcontrolled Peltier device (Juusola and Hardie, 2001).

To preclude poor data biasing our analysis, only high quality stable recordings were used. Such photoreceptors had resting potentials in the dark $<-50 \mathrm{mV}$ and maximum responses to saturating bright pulses $>40$ $\mathrm{mV}$ (WT Canton-S, all mutants and controls). For the used LMCs, the resting potentials were $<-30 \mathrm{mV}$ and maximum responses $>15 \mathrm{mV}$ (WT Canton-S, all mutants and controls). In Calliphora lamina, L1 and L2 generate similar responses, while the responses of L3 are more hyperpolarized, showing the largest off-transients (Uusitalo et al., 1995b). In Drosophila, we have not identified different LMC subtypes, but as L1 and L2 occupy the largest volume most recordings were probably in them. It is also possible that we occasionally record from processes of amacrine cells that share histaminergic input with L2 and L1 cells (Shaw, 1984; Zheng et al., 2006, 2009). However, because the selected recordings to the given stimuli in Drosophila lamina had rather similar hyperpolarizing characteristics, all LMC data were analyzed together. Moreover, attributable to the smaller dimensions of L4 monopolar cell and their nonhistaminergic inputs (Kolodziejczyk et al., 2008), it is unlikely that any of the stable recordings (used in this study) would be from them.

Cells were stimulated at the center of their receptive fields with a high-intensity green-light-emitting diode (Marl Optosource, with peak emission at $525 \mathrm{~nm}$ ). The light stimulus was delivered through a fiber optic bundle, mounted on a rotatable Cardan arm, subtending $5^{\circ}$ as seen by the fly. Its luminance was controlled by neutral density filters (Kodak Wratten), covering a 4 log unit range up to $6 \times 10^{6}$ photons/s (Juusola and Hardie, 2001). Figures show results for $\operatorname{dim}(6000$ photons/s), me$\operatorname{dium}\left(6 \times 10^{5}\right.$ photons $\left./ \mathrm{s}\right)$, and bright luminance $\left(6 \times 10^{6}\right.$ photons $\left./ \mathrm{s}\right)$, corresponding to $\log -3, \log -1$, and $\log 0$. The responsiveness of the cells was tested by repeated presentations of light pulses or naturalistic light intensity series $(10,000$ points/s). Naturalistic stimulus patterns were selected from the van Hateren natural stimulus collection (van Hateren, 1997). Since their luminance was adjusted by placing neutral density filters on the light source, the stimulus sequence retained its contrast constant $(c=\Delta I / I)$.

Voltage responses were amplified by an SEC-10L single-electrode amplifier (NPI Electronic) in current-clamp mode using $15 \mathrm{kHz}$ switching rate. The stimuli and responses were low-pass filtered at $500 \mathrm{~Hz}(\mathrm{Ke}-$ moVBF8), and sampled at 1 or $10 \mathrm{kHz}$. The data were often resampled/ processed off-line at $1-2 \mathrm{kHz}$ for the analysis. Stimulus generation and data acquisition were performed by custom-written Matlab (MathWorks) programs: BIOSYST (Juusola and Hardie, 2001; Juusola and de Polavieja, 2003), with an interface package for National Instruments boards (MATDAQ; H. P. C. Robinson, 1997-2005).

Data analysis. Signal and noise components of photoreceptor voltage responses were estimated both in the time and frequency domains. The signal was obtained from the average of consecutive 1000-ms-long voltage responses to a repeated naturalistic light intensity pattern, and its power spectrum was calculated using Matlab's fast Fourier transform (FFT) algorithm. Only steady-state adapted responses were analyzed; first 10-20 responses were omitted because of their adaptive trends. The noise was the difference between individual responses and the signal, and its power spectra were calculated from the corresponding traces (Juusola et al., 1994). Therefore, for an experiment using $n$ trials (with $n=40-$ 90 ), there was one signal trace and $n$ noise traces. Both signal and noise data chunks were divided into $50 \%$ overlapping stretches and windowed with a Blackman-Harris-term window, each giving seven 250-point-long samples. Thus, we obtained $280-630$ spectral samples for the noise and seven spectral samples for the signal. These were averaged, respectively, to improve the estimates.

A triple extrapolation method (Juusola and de Polavieja, 2003) was used to estimate the rate of information transfer, $R$, of steady-stateadapted photoreceptor voltage responses to naturalistic stimulus, NS. This method, unlike signal-to-noise ratio analysis, requires no assumptions about the signal and noise distributions or their additivity (Juusola and de Polavieja, 2003).

Photoreceptor voltage responses were digitized by sectioning them into time intervals, $T$, that were subdivided into smaller intervals $t=1$ ms. (Only dim luminance data were down-sampled to $125 \mathrm{~Hz}$, giving $t=$ 
$8 \mathrm{~ms}$, which better represented their slow dynamics.) This approach captures 'words' of length $T$ with $T / t$ 'letters.' The mutual information between the response, $s$, and the stimulus is then the difference between the total entropy:

$$
H_{S}=-\sum_{i} P_{S}\left(S_{i}\right) \log _{2} P_{S}\left(S_{i}\right)
$$

and the noise entropy:

$$
H_{N}=-\left\langle\sum_{i=1} P_{i}(\tau) \log _{2} P_{i}(\tau)\right\rangle_{\tau},
$$

where $P_{\mathrm{i}}(\tau)$ is the probability of finding the $i$ th word at a time $t$ from the onset of the trial. This probability $P_{\mathrm{i}}(\tau)$ was calculated across trials to the repeated NS. The values of the digitized entropies depend on the length of the 'words' $T$, the number of voltage levels $v$, and the size of the data file, $H^{T, n \text {,size }}$.

The estimates for the entropy rate, $R_{S}$, and noise entropy rate, $R_{N}$, were then extrapolated from the values of the experimentally obtained entropies to their successive limits, as described by Gonzalez-Bellido et al. (2011).

$$
R=R_{S}-R_{N}=\lim _{T \rightarrow \infty} \frac{1}{T} \lim _{v \rightarrow \infty} \lim _{\text {size } \rightarrow \infty}\left(H_{S}^{T, v, \text { size }}-H_{N}^{T, v, \text { size }}\right) .
$$

The difference between the entropy and noise entropy rates gives then the rate of information transfer (Shannon, 1948; Juusola and de Polavieja, 2003).

\section{Results}

\section{The $d S K$ gene in Drosophila}

$d S K$ is a large gene $(\sim 61 \mathrm{~kb})$ containing 18 exons and produces at least three splice variants (Fig. $1 B$ ), which may encode two distinct protein isoforms: dSK-A and $\mathrm{E}$ (Fig. 1C). Both hypothetical isoforms share the core exons encoding the main functional domains but contain different exons, leading to $\mathrm{N}$ termini of different lengths. dSK is very well conserved (Fig. $1 A$ ) and shows high similarity with its mammalian homologs across the functional regions distinctive of SK channels, including the six transmembrane, $\mathrm{K}^{+}$pore, and calmodulin binding (CAMBD) domains. Both the $\mathrm{N}$ and $\mathrm{C}$ termini show more divergence across species, a feature also seen among different SK genes within the same species (Köhler et al., 1996). Finally, hydropathy tests predict a similar dSK membrane topology as with the mammalian SK orthologs (data not shown).

Based on this structural similarity, we predicted dSK to have conserved functions in the CNS. To study its role in the adult brain, we first aimed to isolate viable mutations in the $d S K$ gene. Given its large size, imprecise excision of transposable P-elements was inefficient in disrupting the $d S K$ gene. Instead, we generated a well defined deletion in $d S K$ using FLP-FRT-mediated recombination (Parks et al., 2004). In this allele $\left(d S K^{-}\right)$, the exons encoding the six transmembrane and CAMBD domains (Fig. $1 B, C$ ) were deleted, and the allele was predicted to be a null. We verified the genomic structure of this deletion by DNA sequencing and PCR amplification (Fig. 1D). Flies homozygous for this allele were fertile and viable.

\section{dSK encodes a slow $\mathrm{Ca}^{2+}$-activated $\mathrm{K}^{+}$current in Drosophila photoreceptors}

Does the fly express any slow $\mathrm{Ca}^{2+}$-activated $\mathrm{K}^{+}$current? In Drosophila larval muscle, two $\mathrm{Ca}^{2+}$-activated $\mathrm{K}^{+}$currents, $I_{\mathrm{CF}}$ and $I_{\mathrm{CS}}$, have been distinguished in conventional voltage-clamp experiments (Gho and Mallart, 1986). The slowpoke mutation (slo) specifically eliminates the fast current, $I_{\mathrm{CF}}$ (Singh and $\mathrm{Wu}$, 1989; Komatsu et al., 1990). No such molecular correlate has been identified for the slow $I_{\mathrm{CS}}$ current. In the eye, one earlier

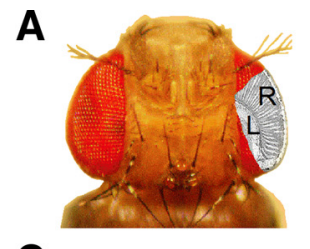

C
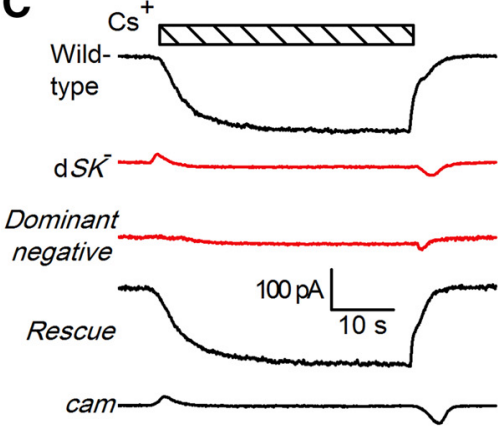

Wild-type $\left(0^{\prime} \mathrm{Ca}^{2+}\right)$

E
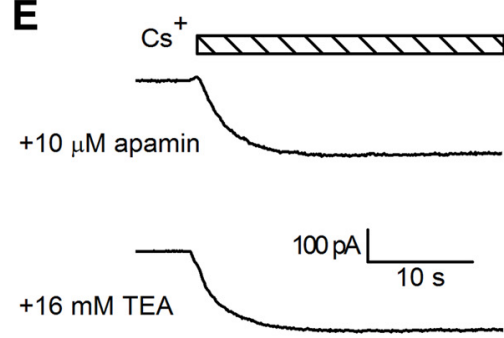

D

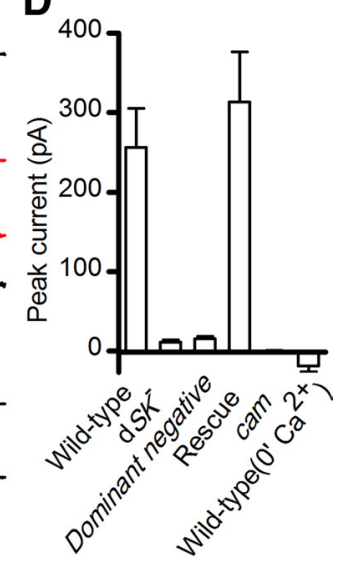

$\mathbf{F}$

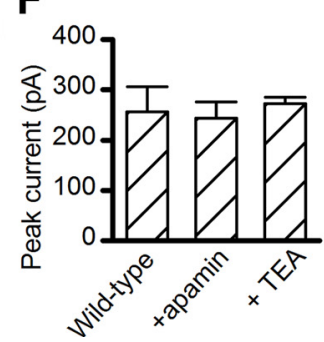

Figure 2. Whole-cell recordings of small-conductance $\mathrm{Ca}^{2+}$-activated $\mathrm{K}^{+}$current, activated by reverse $\mathrm{Na}^{+} / \mathrm{Ca}^{2+}$ exchange in isolated photoreceptors. A, Drosophila head, highlighting the layout of ommatidial arrays. R1-R6 photoreceptor axons link retina (R) to lamina (L). B, Dissociated ommatidium preparation for whole-cell patch-clamping R1-R6 photoreceptors; recording electrode in right. Photoreceptors are disconnected from the lamina network. C, dSK current induced by substituting $\mathrm{CS}^{+}$for extracellular $\mathrm{Na}^{+}$(bar), in WT (in the presence and absence of $\mathrm{Ca}^{2+}$ ), dSK${ }^{-}$, dominant-negative (actingal4/+;UASSKDNmyc), rescue (actingal4/+;UAS-SKmyc), and calmodulin mutant (cam) photoreceptors. Intra-electrode solution contained $20 \mathrm{~mm} \mathrm{Na}{ }^{+}$. All recordings held at $-70 \mathrm{mV}$ and with extracellular solution containing $150 \mu \mathrm{m}$ ouabain. $\boldsymbol{D}$, Peak dSK currents in WT (in the presence, $n=$ 8 , or absence, $n=5$, of $\left.\mathrm{Ca}^{2+}\right), d S K^{-}(n=7)$, dominant-negative $(n=5)$, rescue $(n=7)$, and cam $(n=10)$ photoreceptors. $\boldsymbol{E}$, dSK currents induced by $(s+$ substitution (bar). $\boldsymbol{F}$, The induced dSK current $(\boldsymbol{E})$ was insensitive to the voltage-dependent $\mathrm{K}^{+}$channel blocker, TEA, and apamin (control, $n=8$; TEA, $n=9$; apamin, $n=6$ ).

study reported the existence of a slow $\mathrm{Ca}^{2+}$-activated cationic current in photoreceptors from dissociated ommatidia under voltage-clamp in the whole-cell configuration (Hardie, 1995). This novel conductance was uncovered by increasing intracellular $\mathrm{Ca}^{2+}$ concentration and was distinguished from the lightactivated conductance by being smaller ( $300 \mathrm{pA}$ vs $20 \mathrm{nA}$ ) and trp (transient receptor potential) independent. Later, this current was postulated to be carried by $\mathrm{K}^{+}$(Gu et al., 2005).

To test whether dSK underlies such a current, we evoked the slow $\mathrm{Ca}^{2+}$-activated cationic current in whole-cell recordings from photoreceptors in dissociated ommatidia (Fig. $2 A, B$ ) by artificially driving reverse $\mathrm{Na}^{+} / \mathrm{Ca}^{2+}$ exchange to increase intracellular $\mathrm{Ca}^{2+}$ (Gu et al., 2005; Wang et al., 2005). In this method, the current was induced by substituting $\mathrm{Cs}^{+}$or $\mathrm{K}^{+}$for extracellular $\mathrm{Na}^{+}$while recording with raised intracellular $\mathrm{Na}^{+}(20 \mathrm{mM})$ in the electrode. The $\mathrm{Na}^{+}$-free substitution activated a reverse $\mathrm{Na}^{+} / \mathrm{Ca}^{2+}$ exchange $\left(\mathrm{Ca}^{2+}\right.$ influx for $\mathrm{Na}^{+}$efflux), which 

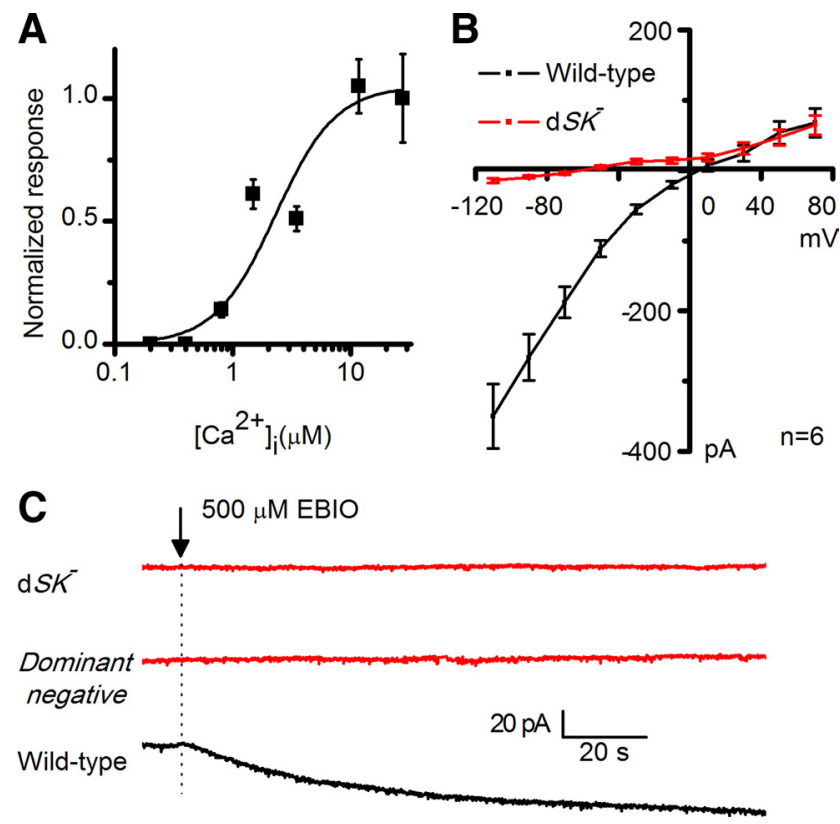

Figure 3. Characterization of the dSK current. $\boldsymbol{A}_{1} \mathrm{Ca}^{2+}$ dependence. Intracellular $\mathrm{Ca}^{2+}$ levels were controlled by using different extracellular $\mathrm{Na}^{+}$concentrations $\left(\mathrm{Na}_{0}\right)$ to manipulate the $\mathrm{Na}^{+} / \mathrm{Ca}^{2+}$ exchanger equilibrium. Predicted intracellular $\mathrm{Ca}^{2+}$ level $\left(\mathrm{Ca}_{\mathrm{i}}\right)$ was calculated from:

$$
\left[C a_{\mathrm{i}}\right]=\left[\mathrm{Ca}_{0}\right] \frac{\left[N a_{\mathrm{i}}\right]^{3}}{\left[\mathrm{Na}_{0}\right]^{3}} e^{\frac{E F}{R T}} .
$$

Internal solution contained 10 or $20 \mathrm{~mm} \mathrm{Na}_{\mathrm{i}}$. External bath contained $40 \mathrm{~mm} \mathrm{~K}^{+}, 85 \mathrm{~mm} \mathrm{Na}^{+}$, $1.5 \mathrm{~mm} \mathrm{Ca}^{2+}$, and $4 \mathrm{~mm} \mathrm{Mg}{ }^{2+}$. SK currents were evoked by rapid perfusion with different $\mathrm{Na}_{0}$ (substituted with Li, which was essentially impermeant) and normalized to maximum currents elicited by $15 \mathrm{~mm} \mathrm{Na}_{0}{ }^{+}$(predicted $\mathrm{Ca}_{\mathrm{i}}{ }^{2+} 28 \mu \mathrm{m}$ with $10 \mathrm{~mm} \mathrm{Na}_{\mathrm{i}}{ }^{+}$at $-70 \mathrm{mV}$ ). Mean \pm SEM on the basis of $n=4-13$ cells per data point with various external $\mathrm{Na}^{+}$concentrations and holding potential of $-70 \mathrm{mV}$. Data were fitted with apparent half-maximal activation $2.9 \mu \mathrm{m}$ and Hill coefficient of 1.34. B, Current-voltage (I/V) relationship of the dSK current induced by reverse $\mathrm{Na}^{+} / \mathrm{Ca}^{+2}$ exchange. WT $(n=7)$ and $d S K^{-}(n=6)$ photoreceptors were subject to voltage steps between $-110 \mathrm{mV}$ and $+70 \mathrm{mV}(20 \mathrm{mV}$ steps) before (control) and after ion substitution ( $\mathrm{Ca}^{+2}$ activation and $\mathrm{dSK}$ induction). The I/V plot was achieved by subtracting control from ion substitution currents at each voltage step. Here, the dSK current was triggered by ion substitution with external $125 \mathrm{~mm} \mathrm{Cs}{ }^{+}$. The internal electrode included TEA. The dSK current shows inward rectification (compare with $d S K^{-}$, red line). C, Activation of dSK current by 1-EBIO. Whole-cell currents were recorded at $-70 \mathrm{mV}$ from WT in response to 1-EBI0 (500 $\mu \mathrm{m}$ ) applied to the bath Ringer's solution containing $40 \mathrm{~mm} \mathrm{KCl}$. A slowly developing inward current of $\sim 50 \mathrm{pA}$, which remains active indefinitely during 1-EBIO perfusion, is evoked in WT photoreceptors ( $n=12$ cells). This conspicuous EBI0-induced current is absent in both $\mathrm{SSK}^{-}$ ( $n=8$ cells) and dominant-negative ( $n=6$ cells) lines.

thereby raised intracellular $\mathrm{Ca}^{2+}$ level (complete $\mathrm{Na}^{+}$substitution, $\mathrm{Ca}^{2+}$ in excess of $10 \mu \mathrm{M}$ at $-70 \mathrm{mV}$ ). In wild-type photoreceptors, using $\mathrm{Cs}^{+}$as charge carrier (SK channels have finite permeability for $\mathrm{Cs}$ ) this procedure activated a large inward current of up to $\sim 250 \mathrm{pA}$ (Fig. 2C). This conspicuous current was also permeable to $\mathrm{K}^{+}$(Fig. 3) (but not $\mathrm{Li}^{+}$: data not shown), was maximally activated within seconds after reverse $\mathrm{Na}^{+}$/ $\mathrm{Ca}^{2+}$ exchange, and inactivated upon $\mathrm{Na}^{+}$return. Furthermore, the current was $\mathrm{Ca}^{2+} /$ calmodulin-dependent as it was absent either when extracellular $\mathrm{Ca}^{2+}$ was removed or in cam flies lacking calmodulin (Fig. $2 C, D$ ). The $\mathrm{Ca}^{2+}$ dependence, quantified by using different concentrations of $\mathrm{Na}_{\mathrm{o}}$ to manipulate the $\mathrm{Na}^{+}$/ $\mathrm{Ca}^{2+}$ equilibrium, showed an apparent $\mathrm{EC}_{50}$ of $2.9 \mu \mathrm{M}$ (Fig. $3 A$ ). Importantly, the current was abolished in $d S K^{-}$photoreceptors and fully restored when a UAS-SKmyc rescue construct was expressed in the $d S K^{-}$-null background (Fig. $2 C, D$ ). We propose that this novel slow $\mathrm{Ca}^{2+}$-activated, $\mathrm{K}^{+}$- and $\mathrm{Cs}^{+}$-permeable current isolated from Drosophila photoreceptors is the electrophysiological correlate of $d S K$.

Although the dSK channel is voltage insensitive, its currentvoltage relationship is not linear, exhibiting increased conductance with hyperpolarization (Fig. 3B). This inward rectification, also shown earlier (Hardie, 1995), is shared with its mammalian homologs, suggesting a similar voltage-dependent block by intracellular divalent cations. SK channels are pharmacologically distinguished from BK channels by their insensitivity to low concentrations of external tetraethylammonium or TEA (Blatz and Magleby, 1986). Similarly, the dSK current was not affected by TEA application (Fig. 2E,F). Mammalian SK channels are enhanced by the agonist 1-Ethyl-2-benzimidazolinone (1-EBIO) and blocked by apamin, a honeybee venom toxin (Blatz and Magleby, 1986; Pedarzani et al., 2001). In dissociated photoreceptors, 1-EBIO induced an SK current in wild-type but not in $d S K^{-}$-null photoreceptors (Fig. 3C). However, the dSK current was not altered by apamin treatment, suggesting that dSK is apamin insensitive (Fig. 2E,F). Two amino acid residues, $243 \mathrm{~S}$ and 340D have been shown to be important for apamin binding in SK2 channel (Ishii et al., 1997; Nolting et al., 2007). Interestingly, neither residue is present at the equivalent positions (191K and $286 \mathrm{H}$ ) in the dSK primary amino acid sequence (Fig. $1 \mathrm{~A}$ ).

In summary, Drosophila photoreceptors express a dSKdependent $\mathrm{Ca}^{2+}$-activated $\mathrm{K}^{+}$current, which bears high resemblance to the mammalian SK current, pointing toward conserved role(s) for this conductance in neurons. We also speculate that dSK is the molecular correlate of the apamin-insensitive slow $\mathrm{Ca}^{2+}$-activated $\mathrm{K}^{+}$current $\left(I_{\mathrm{CS}}\right)$ recorded at the larval muscle and whose identity has long been unknown (Gho and Mallart, 1986; Singh and $\mathrm{Wu}, 1989$ ).

\section{dSK is required for normal photoreceptor light response}

To test the contribution of dSK channel to the photoreceptor voltage output in vivo, we performed intracellular recordings from $d S K^{-}$null photoreceptors and their wild-type counterparts (Fig. $4 \mathrm{~A}$ ) to intensified light flashes (Fig. $4 B, C$ ). Both sets of photoreceptors responded with graded transient depolarizations, covering similar ranges (Fig. $4 B, C$ ). However, $d S K^{-}$photoreceptors showed accelerated kinetics; their responses reached peak amplitudes faster and recovered to resting potential earlier (Fig. 4B,C). Here, we recall that the voltage output of fly photoreceptors constitutes a complex convolution of light current (Hardie and Raghu, 2001), light-insensitive membrane filtering and feedbacks from their neuronal neighbors (Shaw, 1984; Weckström and Laughlin, 1995; Juusola and Hardie, 2001; Zheng et al., 2006).The $d S K$ mutation could, therefore, affect any or all of these mechanisms, leading to the observed fast responses.

To exclude the possibility that $d S K$ deletion could lead to developmental defects and altered photoreceptor morphology, we examined eye sections of dark-reared flies. Both wild-type and $d S K^{-}$eyes consisted of highly ordered units or ommatidia, which had normal photoreceptors with intact rhabdomeres (Fig. $4 D, E$, respectively). However, prolonged light exposure results in minor changes in photoreceptor integrity (data not shown). To test whether the dSK deletion could potentially affect the properties of the phototransduction machinery, leading to altered light-induced currents, we assessed quantum bumps (elementary responses to single-photon absorptions; Fig. $4 \mathrm{~F}$ ) and impulse responses (Fig. $4 \mathrm{H}$ ) to light flashes using whole-cell recordings from R1-R6 photoreceptors in dissociated ommatidia (Hardie et al., 1991). In this preparation, the dissociated photoreceptors lack axonal terminals and thus any synaptic feed- 
back. Bump waveforms in $d S K^{-}$photoreceptors were indistinguishable from those of the wild-type photoreceptors (Fig. 4F, G). Furthermore, macroscopic responses to increasing light intensities were similar in wild-type and mutant photoreceptors and shared the same kinetics (Fig. 4H,I). These data suggest that neither photoreceptor morphology nor the phototransduction machinery is affected by the $d S K$ deletion.

The faster responses in $d S K^{-}$photoreceptors could be caused by faster charging through the photo-insensitive membrane, if the membrane bandwidth is limiting. In the Diptera, species with fast or slow responding photoreceptors exploit different combinations of $\mathrm{K}^{+}$conductances to tune the photoreceptor membrane to match the fly's visual ecology (Weckström and Laughlin, 1995). In the absence of dSK, an increase in compensatory $\mathrm{K}^{+}$conductances could, for example, lower the membrane input resistance, and thus its time constant, helping to explain the observed fast responses in $d S K^{-}$photoreceptors. To test this possibility, we again used whole-cell recordings from photoreceptors in dissociated ommatidia (which lack axons) (cf. Fig. 2B). Interestingly, compared with wild-type, mutant cells had $\sim 25 \%$ reduction in Shaker $\left(I_{\mathrm{A}}\right)$ current but normal slow delayed rectifier $\left(I_{\mathrm{KS}}\right.$, or Shab) current (data not shown). In addition to the absence of dSK current, the observed decrease in $I_{\mathrm{A}}$ current should, all things being equal, lead to higher membrane resistance and slower responses. Consequently, the photo-insensitive membrane properties together with the normal phototransduction machinery suggest that the observed faster intracellular responses in mutant photoreceptors reflect a role for dSK in later membrane processes at the photoreceptor axon/synapse.

\section{dSK is highly enriched in the optic lobe}

To examine the expression pattern of dSK in the fly brain, we generated a rabbit anti-dSK polyclonal antibody against the $\mathrm{N}$-terminal domain of the protein. Using Western blot analysis on adult head extracts, we detected two bands from wild-type flies that were absent from $d S K^{-}$, confirming that the latter is a null allele (Fig. 5A). While we cannot rule out the presence of other dSK isoforms from other body parts, our data suggest that there could be two dSK isoforms expressed in adult heads. Alternatively, the double bands could correspond to one dSK isoform and its post-translationally modified form.

Immunostaining for dSK in the adult fly brain revealed a broad expression throughout several CNS regions (Fig. 5B). Interestingly, higher expression levels were observed in the lamina and medulla neuropils of the optic lobe with a weaker expression in the retina. Despite the weak retinal expression, it must be functional within the retina since dSK activity can be detected in isolated photoreceptors. In the lamina, dSK showed a remarkable
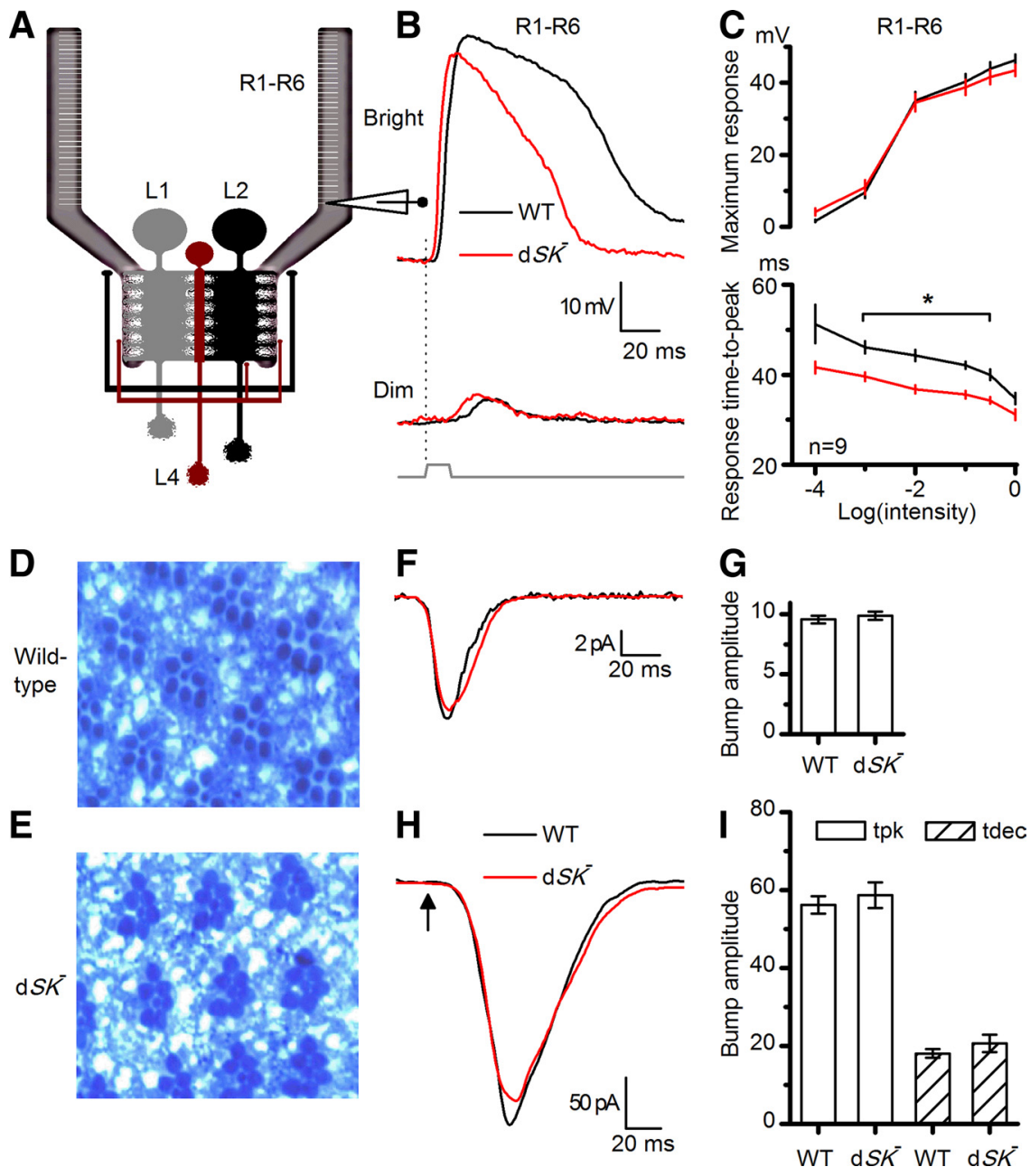

Figure 4. $d S K^{-}$photoreceptors have faster responses and intact phototransduction. $\boldsymbol{A}$, In vivo recordings from $\mathrm{R} 1-\mathrm{R} 6 \mathrm{phot0}$ receptors, connected to the lamina network; schematic highlights feedback connections ( $22 / A C$ and L4) to R1-R6 axon terminals. $B$, Voltage responses of WT and $d S K^{-}$photoreceptors to a bright and a dim $10 \mathrm{~ms}$ pulse in the dark. $d S K^{-}$responses rise and decay means of 9 cells. $C$, Voltage ranges of WT and $d S K^{-}$are similar, but response time-to-peak is faster in $d S K^{-} .{ }^{*} p<0.020$, $t$ test. $\boldsymbol{D}$, Retinal cross section $(1 \mu \mathrm{m})$ of WT eye. $\boldsymbol{E}$, Retinal cross section $(1 \mu \mathrm{m})$ of $d S K^{-}$eye shows intact morphology. $\boldsymbol{F}$, Whole-cell recordings from dissociated photoreceptors. WT and $d S K^{-}$quantum bumps (average of $>30$; aligned by rising phase) are indistinguishable. $\mathbf{G}$, Bump amplitude from $\boldsymbol{F}: \boldsymbol{n}=4-9$ cells. $\boldsymbol{H}$, Macroscopic responses to brief flashes (5 ms; arrow) containing $\sim 75$ effective photons were similar in $d S K^{-}$and WT.

colocalization with Bruchpilot (Fig. 5C), a marker of presynaptic active zones (Wagh et al., 2006), highlighting a potential role for $\mathrm{dSK}$ in neurotransmitter release at the first visual network. In the central brain, dSK was present in several neuronal processes with strong expression in the projection neuron axons connecting the antennal lobe to the mushroom bodies (Fig. 5B). This strikingly high abundance in the visual and olfactory systems points toward a conserved role for dSK in sensory processing and olfactory learning.

In the lamina, axon terminals of R1-R6 photoreceptors make histaminergic feedforward synapses with the primary visual interneurons: the LMCs (L1-L3) and the ACs. In return, photoreceptor terminals receive direct feedback inputs from the L2 and AC interneurons via ligand-gated feedback synapses (Meinertzhagen and Sorra, 2001). Within the lamina synaptic region, dSK could either be pre- and/or postsynaptic (Fig. 5C). Colocalization with chaoptin, a specific membrane marker of photoreceptors cells (Reinke et al., 1988; Van Vactor et al., 1988), indicated that dSK is highly abundant in photoreceptor axons (Fig. 5D,H). The AC and L2 interneu- 
A
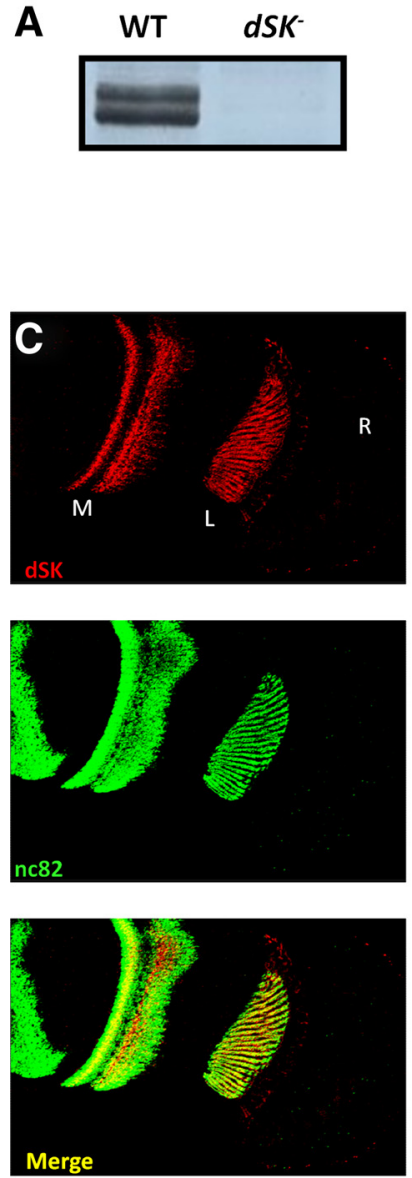
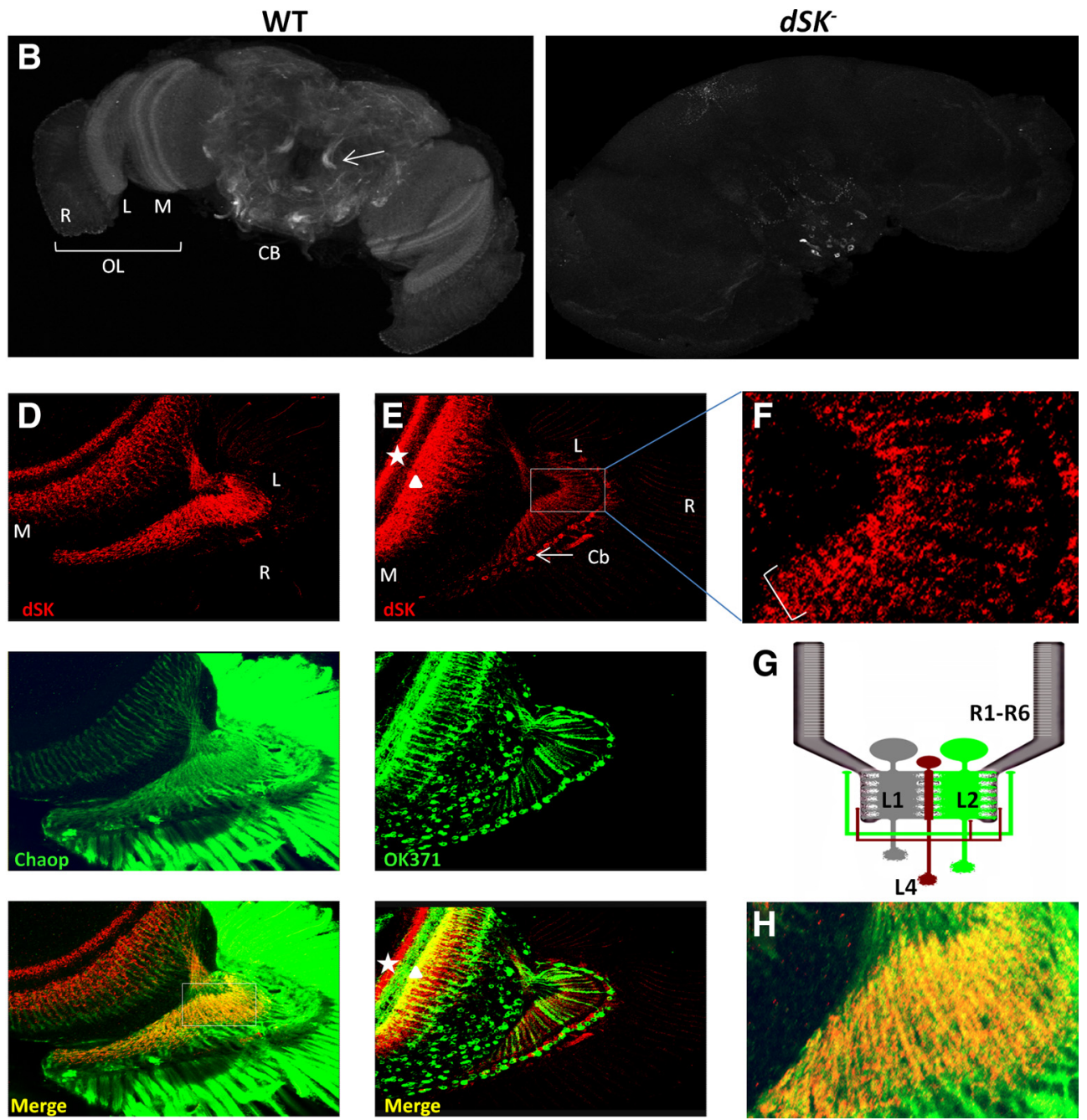

Figure 5. dSKis expressed in adult optic lobes. A, Anti-dSK antibodies recognize two membrane proteins from WT fly heads, absent in dSK $^{-}$fly heads. Post-translationally modified isoforms cannot be ruled out. $\boldsymbol{B}$, Projection view of WT and $d S K^{-}$adult brains stained with anti-dSK antibodies. dSK is absent in $d S K^{-}$brains, confirming the antibody specificity. Arrow indicates projection neurons in the central brain (CB). OL, Optic lobe; R, retina; L, lamina; M, medulla. C, Single confocal section view of the optic lobe stained with anti-dSK (red) and anti-Bruchpilot (green) antibodies. dSK highly localizes to neuropils of the optic lobe. $\boldsymbol{D}$, Confocal section through an optic lobe labeled with photoreceptor membrane marker, chaoptin (Chaop, green). dSK (red) labels photoreceptor axons at lamina (L). $\boldsymbol{E}$, Confocal section through an OK371-Gal4/+;UAS-mCD8-GFP/ + brain at the optic lobe level. GFP (green) labels glutamatergic neurons. In lamina, dSK (red) localizes to nonglutamatergic neuron bodies (Cb) (arrow). In medulla, dSK labels nonglutamatergic (star) and possibly also some glutamatergic (triangle) neurons. $F$, A magnification of the squared area in $E$. Bracket highlights dSK-positive lateral branching at proximal lamina, characteristic of L4 neurons. G, A schematic portraying lamina connections. ASK is absent from glutamatergic direct feedback neurons, L2/AC (green), while expressed in L4 neuron (red), which makes lateral feedback connections (also in $\boldsymbol{F}$ ) into R1-R6 and L2 neurons (f. $\boldsymbol{E}$ ). $\boldsymbol{H}$, A magnification of the squared area in $\boldsymbol{D}$, bottom. In the lamina, dSK (red) highly localizes to photoreceptor axons stained with Chaoptin (green).

rons were previously shown to be glutamate positive (Sinakevitch and Strausfeld, 2004; Kolodziejczyk et al., 2008; Raghu and Borst, 2011). We decorated glutamatergic neurons in the adult brain with a membrane GFP (UAS-mCD8GFP) using OK371-Gal4, an enhancer trap line inserted in the Drosophila vesicular glutamate transporter (DVGLUT) gene (Mahr and Aberle, 2006). Although dSK showed expression in some lamina neurons, those neurons were nonglutamatergic (Fig. $5 E$ ). In the proximal lamina, some of them formed lateral branches (Fig. $5 F$ ), characteristic of cholinergic L4 monopolar cells (Fischbach and Dittrich, 1989; Kolodziejczyk et al., 2008). In the medulla, the dSK antibody labeled axonal terminals of nonglutamatergic and, possibly, some overlapping glutamatergic neurons (Fig. $5 E$ ). The terminals of cholinergic L4s occupy layer M4, while those of L5 monopolar cells spread delicately into layers M1 and M2 (which also contain L1 and L2 terminals, respectively; marked by triangle) and more densely into M5 (Fischbach and Dittrich, 1989). The dense dSK expression in probable M4-M5 layers (marked by star) implies that L4 together with L5 or L1 monopolar cells, or all of them, were expressing dSK.
To summarize, dSK is highly enriched at the lamina synaptic network where it localizes to photoreceptor axons and a group of nonglutamatergic monopolar cells; including L4s, which receive indirect input from R1-R6 photoreceptors through the lamina network but form feedback synapses both to L2 monopolar cells and R1-R6 terminals (Fig. 5G) (Meinertzhagen and O'Neil, 1991). Nonetheless, since dSK is absent from the major AC feedback interneurons, and also from L2 monopolar cells, the direct synaptic feedbacks to R1-R6 photoreceptors axons should be less affected. dSK expression pattern, therefore, provides an additional evidence that dSK contributes to photoreceptor voltage response by finetuning feedforward output and/or feedback input at photoreceptor axons.

dSK contributes to the photoreceptor axon membrane potential in the dark

Given this high expression at the layer of the first visual synapse, $\mathrm{dSK}$ channels may play a role in facilitating sensitivity regulation 
by counteracting $\mathrm{Ca}^{2+}$ influx in this network where the feedforward and feedback crosstalk tightly adjusts the potentials of the cells (Zheng et al., 2006). In the dark, LMCs and AC receive less feedforward inputs and therefore are more depolarized. This, in return, will adjust the photoreceptor's potential to more positive values (compared with dissociated photoreceptors) due to increased feedback inputs from the interneurons. Consequently, dSK removal from synapses in this network would disrupt its intricate balance in the dark, altering electrical properties of photoreceptors. To test this hypothesis, we performed in vivo intracellular recordings from darkadapted wild-type and $d S K^{-}$photoreceptors (Fig. 6A-C). Strikingly, voltage responses evoked by negative current steps revealed that input resistance of the $d S K^{-}$ photoreceptors was significantly decreased relative to the wild-type cells (Fig. $6 B, C$ ). The decrease in input resistance was also accompanied with a more depolarized resting potential in the dark (Fig. 6C). These findings strongly suggest an increase in depolarizing conductances in/into $d S K^{-}$ photoreceptor axons. While it is likely to contribute to the more positive value of the $d S K^{-}$photoreceptor resting potential, the reduced Shaker conductance, together with removal of dSK conductance, would be expected to result in higher resistance at rest. We, therefore, conclude that the observed lowered input resistance is likely to result from increased axonal depolarizing conductance and not from excess $\mathrm{K}^{+}$ conductance (which would hyperpolarize the membrane appreciably relative to wild-type).

Overall, these data support a role for dSK in fine-tuning photoreceptor resting potential and its input resistance. Additionally, the decrease in photoreceptor's input resistance explains, at least partially, the faster light responses seen in the intracellular recordings from the mutant photoreceptors (Fig. 4B, C). Furthermore, our expression data, the normal light-induced current, and the photo-insensitive membrane dynamics strongly suggest that this lowered input resistance and the consequent fast $d S K^{-}$photoreceptor responses are attributable to a change in photoreceptor's axonal inputs and/or output in the dark.

\section{dSK mediates light-dependent sensitivity regulation at the lamina network}

Blocking mammalian SK channels has been shown to enhance activity-dependent synaptic transmission and plasticity, suggesting a role for SK in coupling to and negatively regulating synaptic $\mathrm{Ca}^{2+}$ sources (Faber et al., 2005; Ngo-Anh et al., 2005). To test the effect of dSK removal on light-dependent activity in the lamina network, we first recorded intracellular voltage responses of photoreceptors in wild-type and $d S K^{-}$flies to prolonged light pulses. We discovered that in most recordings the responses of mutant photoreceptors oscillated distinctively at $40 \mathrm{~Hz}$ and $70 \mathrm{~Hz}$ during and after prolonged light adaptation (LA) (Fig. 6D,E). Notably, the oscillations depended on light history (cf. before and after LA) and were boosted at lower potentials (cf. during and after LA) that should drive stronger LMC/AC feedbacks into photoreceptors (Zheng et al., 2006). Furthermore, in the dark, when their output showed no oscillations, $d S K^{-}$photoreceptors were noisier than their wild-type counterparts (Fig. 6E, before LA); probably due to a barrage of depolarizing synaptic inputs into their axons, leading to the observed low input resistance (Fig. 6C). Nonetheless, since these recordings were performed at the level of photoreceptor somata, the observed dynamics were likely to underestimate the processing taking place at the axon terminals.

We next recorded intracellularly from postsynaptic LMCs in vivo (Fig. $7 A$ ). Although both wild-type and $d S K^{-}$LMCs re- 
A
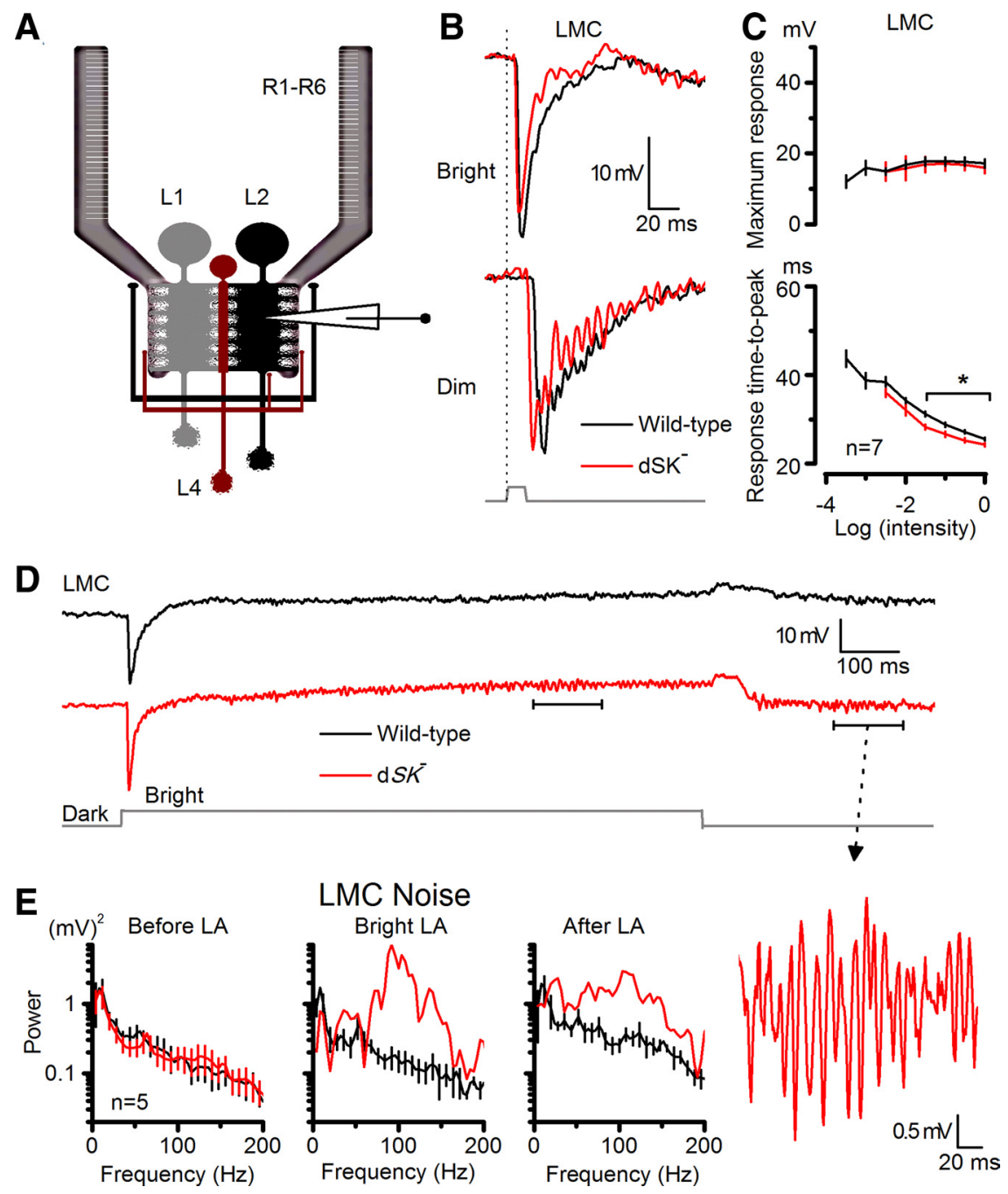

Figure 7. dSK fine-tunes synaptic transmission to LMCS. $A$, Recordings from LMCs in intactlamina; feedback connections (L2/AC and L4) to R1-R6 photoreceptor axon terminals are highlighted. $\boldsymbol{B}$, Voltage responses of WT and dSK ${ }^{-}$LMCs to a bright and a dim $10 \mathrm{~ms}$ pulse in the dark. $d S K^{-}$responses fall and rise faster. $C$, Respond ranges of WT and $d S K^{-}$are similar, but time-to-peak to bright pulses is faster in $d S K^{-}\left(p<0.019 ; t\right.$ test). $\boldsymbol{D}, 0$ scillations in $d S K^{-} \mathrm{LMCs}$ are experience dependent. Voltage responses of WT and $d S K^{-}$before, during, and after bright light adaptation. Inset, An oscillating response magnified. $\boldsymbol{E}$, Noise power spectra of corresponding epochs in $\boldsymbol{D}, W T$ and $d S K^{-}$LMCs, during and after light adaptation. Oscillations occur at $\sim 100 \mathrm{~Hz}$, at higher frequencies than in $d S K^{-}$R1-R6 (Fig. 6E). Mean \pm SEM is shown.

sponded to light flashes with graded hyperpolarizations of similar sizes, the responses of mutant LMCs were significantly faster; thus the LMCs encoded faster presynaptic kinetics (Fig. $7 B, C$ ). Interestingly, $\sim 40 \%$ ( 3 of 7 ) of $d S K^{-}$LMCs showed oscillating responses. The oscillations were exacerbated at dim conditions (Fig. $7 B$ ), where the gain of both the photoreceptor output synapses and synaptic feedbacks is the highest (Juusola et al., 1995; Zheng et al., 2006), thereby leaving the lamina network most perturbable to intrinsic activity. Longer recordings indicated that the oscillations in mutant LMCs carried different frequencies during and after light adaptation (Fig. $7 D, E$ ). Furthermore, compared with photoreceptors, these oscillations were larger and faster, peaking at $\sim 100 \mathrm{~Hz}$ (Fig. $7 E$ ); thus, the network transferred stimulus energy to higher output frequencies. Nonetheless, in both mutant R1-R6s and LMCs, the oscillations varied with the light history (cf. oscillations before and after LA in Fig. 7E), showing clear activity-dependency. Extracellular recordings (electroretinograms or ERGs) showed that while they maintained normal amplitudes, $d S K^{-}$responses exhibited occasional oscillations near resting potential after cessation of light stimulus (data not shown); again reflecting oscillatory network responses that occur at low potentials when feedback synaptic input is highest. Thus, these findings provide further evidence that dSK channels oppose intracellular $\mathrm{Ca}^{2+}$ increases, which are expected to peak during and immediately after light changes, and by this sensitivity control improve packaging of neural messages within the limited signaling bandwidth of the synapses.

\section{dSK role in photoreceptor adaptation and coding}

So far our results have shown that removal of the dSK channel from the synaptic microcircuits between photoreceptors and LMCs leads to activity-dependent oscillations in the lamina network. These perturbations were accompanied with altered response properties of photoreceptors, including decrease in their input resistance, more depolarized resting potentials and faster lightinduced voltage responses. Next, we asked to what degree these changes, many of which mimicked electrophysiological effects of light-adaptation (Klöcker et al., 2001; Nikolaev et al., 2009), affected the adapting properties and signaling performance of $d S K^{-}$photoreceptors.

Our experiments were designed to quantify how well wild-type and $d S K^{-}$ photoreceptors could encode different light patterns (i.e., their intensity resolution) over a wide range of luminances. The photoreceptors were stimulated by repeatedly presenting naturalistic time series of contrasts, collected from natural environments (van Hateren, 1997), while recording their intracellular voltage responses. Consistent with the results of the light pulse experiments, the responses of $d S K^{-}$photoreceptor mostly oscillated at dim stimuli (Fig. 8A), during which the gain of feedforward and feedback synapses is high (Zheng et al., 2006). However, some recordings lacked oscillations and many oscillated only sporadically; in some cases, oscillations died out during stimulation. All these observations underline the variable dynamic nature of gain regulation in the lamina network.

Interestingly, during the stimulation, the mean output of mutant photoreceptors adapted to the tested stimulus luminances much faster than that of wild-type photoreceptors (Fig. $8 B$ ), suggesting that the lamina network was tonically driving them to elevated states of activity, in tune with their lower input resistance (cf. Fig. 6B). However, early in the adaptation phase, as a sign of limited gain control, the dynamic range of $d S K^{-}$photoreceptors contracted significantly (Fig. 8C). Then, instead of desensitizing to the new stimulus luminance, as wild-type photoreceptors do, most $d S K^{-}$photoreceptors sensitized to it (6 of 8 cells); their responses grew larger as the contrast pattern was repeated, reaching a relative steady-state in $\sim 12 \mathrm{~s}$ of bright naturalistic stimulation. This recovery rate $(\tau \approx 6 \mathrm{~s})$ matches the rate of network 
adaptation in wild-type LMC output ( $\tau=$ 1-7 s), which improves temporal representation of similar stimuli (Zheng et al., 2009), suggesting that inputs from the lamina network might be amplifying $d S K^{-}$photoreceptor output over time. After removing the first 20 responses with clear adapting trends, the photoreceptor signal was estimated by averaging the rest of the responses (Fig. 8D), and the photoreceptor noise was taken as the difference between individual traces and the signal. Their power spectra indicated that, apart from the dimmest stimulation (cf. Fig. $8 \mathrm{~A}$ ), once steady-state adapted, $d S K^{-}$photoreceptors produced larger responses to fast light changes than wild-type cells, allocating more power at higher output frequencies $(>50 \mathrm{~Hz}$; Fig. $8 E$ ), but that these fast responses were more variable (Fig. $8 F$ ). Because oscillations in voltage responses of $d S K^{-}$photoreceptors were sporadic or died out during the repeated stimulation, they contributed relatively little on this analysis (Fig. 8D), which thus can underestimate their real impact. In fact, we expect the oscillations and reduced gain control of $d S K^{-}$photoreceptors at lightdark transitions to blur vision more when a mutant fly locomotes in its natural environment, as this requires even more demanding spatiotemporal gain changes in the lamina network. Nonetheless, here the striking finding was that the variable signal and noise dynamics of wild-type and $d S K^{-}$photoreceptors provided unexpectedly similar information captures of naturalistic stimuli over a large luminance range, saturating at $\sim 300 \mathrm{bits} / \mathrm{s}$ at bright stimulation (Fig. 8G). These results highlight the inherit robustness of encoding in the lamina network (Niven et al., 2003; Vähäsöyrinki et al., 2006; Zheng et al., 2006).

Cellular localization of dSK functions at the first visual layer

Both light-dependent oscillations and lightindependent electrophysiological changes in the lamina network indicate suboptimal sensitivity regulation in photoreceptors and/or interneurons in $d S K^{-}$flies, suggesting dSK has a direct role in synaptic communication as an adaptive damper. To further map dSK's contribution to the lamina network, we used the UAS/GAL4 system to inactivate dSK in a cell-specific manner. Taking advantage of the fact that a functional SK channel is tetramer of four $\alpha$-SK subunits, we designed a dominant-negative dSK subunit (UAS-SKDNmyc) in which the $\mathrm{K}^{+}$pore "GYG" was mutated into "AAA." When expressed in a wild-type background, this subunit completely suppressed the dSK current induced from photoreceptors using either the ion exchange protocol (Fig. 2C,D) or EBIO in dissociated ommatidia (Fig. 3C).
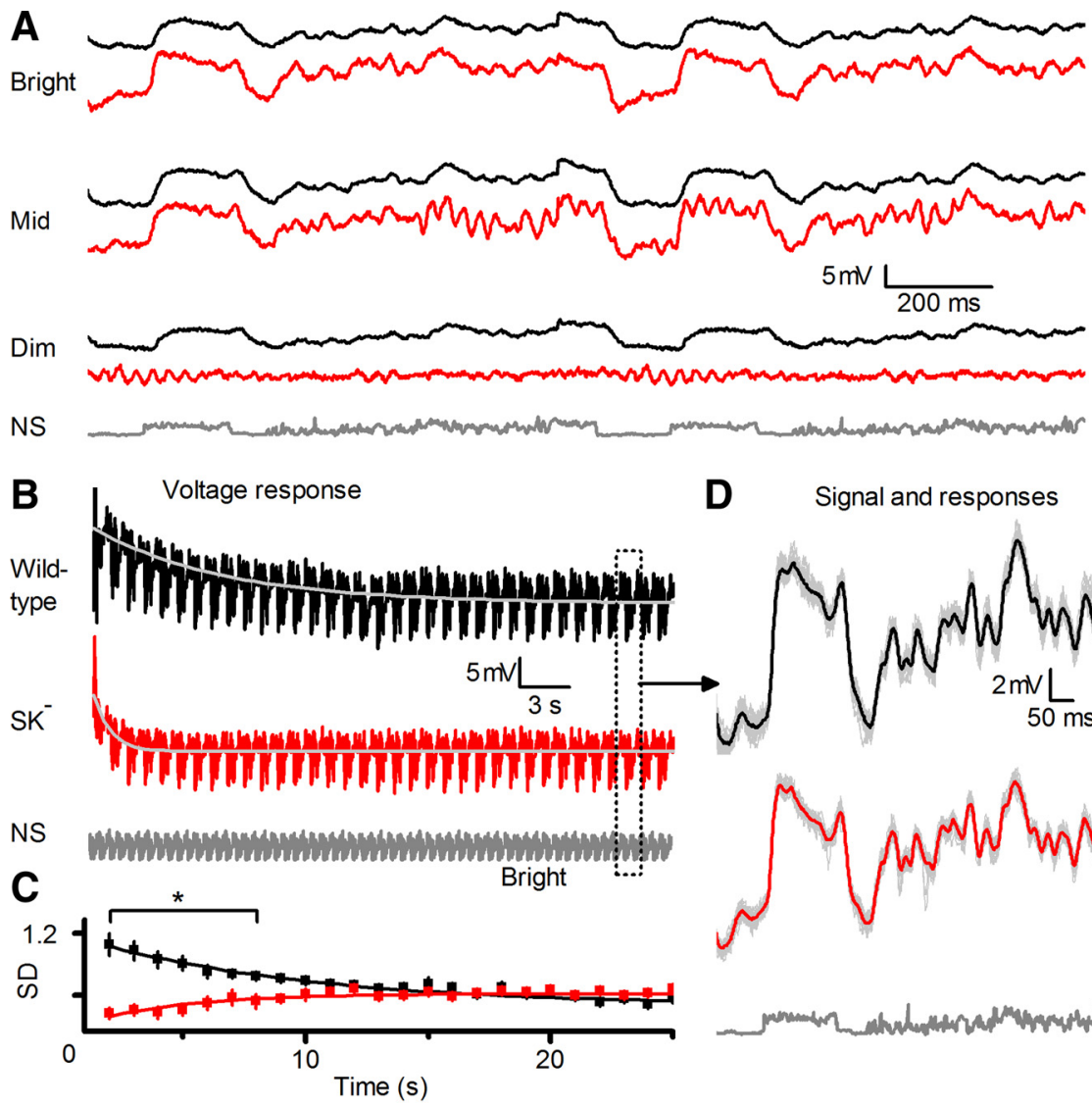

D Signal and responses
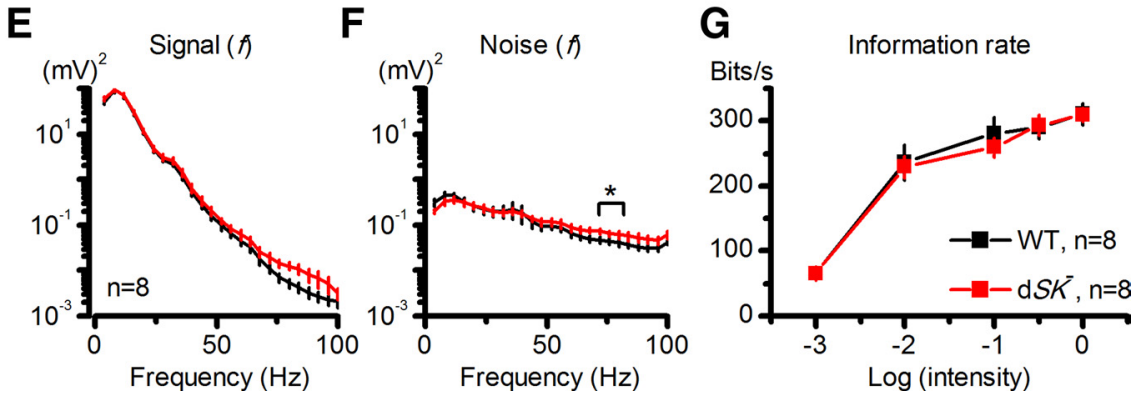

Figure 8. $d S K^{-}$photoreceptors show fast but inefficient adaptation to dark-light transitions, yet their rate of information transfer appears normal. $\boldsymbol{A}$, Voltage responses of $d S K^{-}$photoreceptors oscillate sporadically during NS at dim and middle luminances, but rarely at bright luminances. $\boldsymbol{B}$, Responses of R1-R6 photoreceptors to a repeated bright naturalistic stimulation. Mean $d S K^{-}$photoreceptor output adapted to a steady-state level significantly faster than WT $\left(\tau_{\mathrm{SK}}=1.2 \pm 0.2 \mathrm{~s} ; \tau_{\mathrm{WT}}=6.2 \pm 1.8 \mathrm{~s}\right.$; mean \pm SEM; $p<0.017, n=10$ cells). Dotted window, Repeated $1 \mathrm{~s}$ patterns. C, Dynamic range (SD) of $d S K^{-}$photoreceptor output was significantly reduced at the dark-light transition ( $p<0.024, t$ test), but recovered (sensitized) to the WT level in $\sim 12$ $\mathrm{s}\left(\tau_{\mathrm{SK}}=6.3 \pm 2.8 \mathrm{~s}\right)$. In contrast, WT photoreceptor output contracted (desensitized) and slower ( $\tau_{\mathrm{WT}}=15.4 \pm 4.6 \mathrm{~s}$; mean \pm $\mathrm{SD}, n=6$ ). $\boldsymbol{D}$, The signals (mean responses; black and red) and individual voltage responses (gray) of 8 photoreceptors after steady-state adaptation (first 20 responses omitted). $\boldsymbol{E}$, Signal power spectra: $d S K^{-}$photoreceptors generate larger responses to fast light changes $(70-100 \mathrm{~Hz})$ than WT (mean \pm SEM). $\boldsymbol{F}$, Noise power spectra: $d S K^{-}$photoreceptors are noisier at high frequencies (mean \pm SEM); i.e., at $72 \pm 4 \mathrm{~Hz}(p=0.024)$. G, Information transfer rate estimates: $d S K^{-}$photoreceptor output shows encoding capacities similar to those of WT at all tested luminances (mean \pm SD).

We targeted this dominant-negative dSK subunit to the R1-R6 cells using the eye-specific longGMR-GAL4 driver. Intracellular recordings from photoreceptors expressing a dominantnegative dSK subunit showed accelerated response kinetics, approaching those seen in $d S K^{-}$photoreceptors (Fig. 9). However, no light-dependent oscillations were detected in such photoreceptors, although their conductance deficits match those of $d S K^{-}$photoreceptors (Figs. 2, 3C), suggesting that oscillations seen in mutant photoreceptors and LMCs might result from a less adequately tuned network. This view is consistent with the dSK 


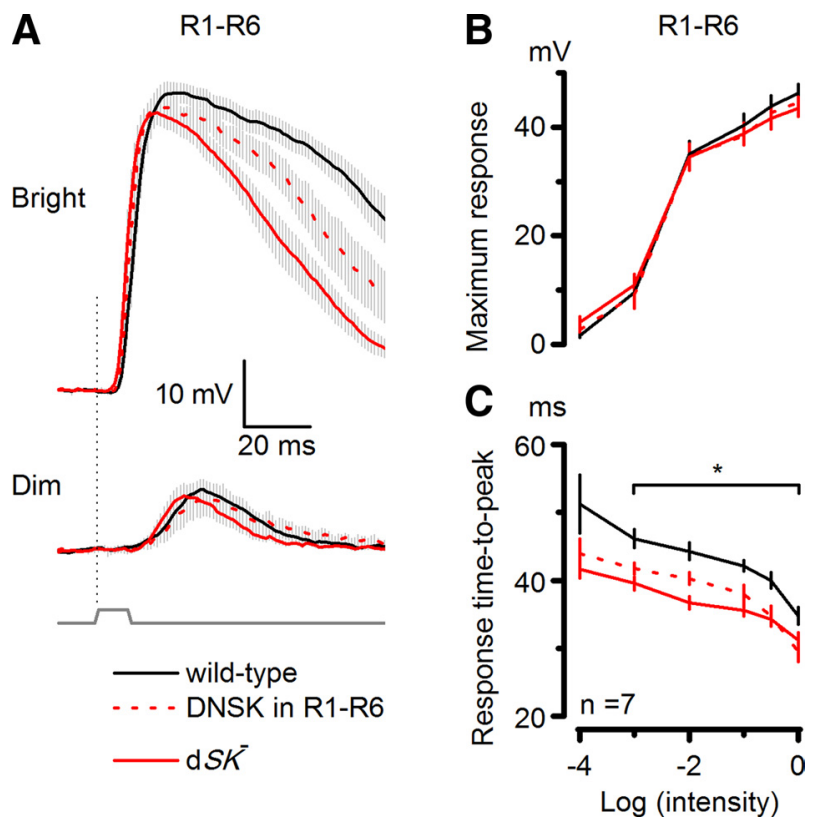

Figure 9. Photoreceptors expressing a dominant-negative dSK subunit have faster light responses. $\boldsymbol{A}$, Voltage responses of WT, $d S K^{-}$, and photoreceptors with specific dSK dominantnegative expression (red dashed line, DNSK in R1-R6) to a bright and a dim 10 ms pulse in the dark. $d S K^{-}$and DNSK in R1-R6 responses rise and decay significantly faster than WT. $\boldsymbol{B}$, Voltage ranges of all genotypes are similar. $\boldsymbol{C}$, Time-to-peak of the responses is as fast in photoreceptors expressing a dominant-negative dSK subunit as in $d S K^{-}$photoreceptors when compared with wild type ( $p<0.0184, n=7$ cells; Student's $t$ test). The genetically altered cells have faster responses over most of the tested light intensities.

immunostaining results (Fig. 5), which suggested that in $\mathrm{dSK}^{-}$ mutants, glutamatergic feedbacks from L2 and AC interneurons to photoreceptor axons should function normally, while some nonglutamatergic contacts in their lamina might not (including those of L4 LMCs). In clear contrast, in flies, where photoreceptor axons express functionally impaired dominant-negative dSK, the lamina is otherwise normal, and so better equipped to compensate this loss in neuronal function (Fig. 9) by tuning its network functions. Thus, oscillations in mutant cells probably arose from improper control of synaptic gain in $d S K^{-}$LMCs, and/or possibly in other interneurons.

\section{Discussion}

The essential characteristic of the Drosophila lamina is its massively parallel synaptic connections (Meinertzhagen and O'Neil, 1991), which, similar to our retina, supposedly evolved to reliably process and redistribute information about environmental regularities to multiple retinotopic pathways (Barlow, 1961). Its robust design can also withstand mutations or damage without losing much of its encoding performance (Zheng et al., 2006). Indeed, we discovered that many detriments of missing dSK channels appeared to have been compensated homeostatically by network functions; most likely by tuning synaptic currents. Nonetheless, this compensation, which disrupted the photoreceptor membrane properties, accelerating responses and some aspects of their adaptation, was suboptimal. Consequently, we could establish that dSK channels in wild-type mediate activitydependent inhibition in the lamina network, preventing responses from oscillating while its synapses operate with high gain; presumably to curb the costs of noise (van Hateren, 1992) and energy (Laughlin et al., 1998). These results concur with the view that dSK current counteracts light-induced $\mathrm{Ca}^{2+}$ increases, which drive transmitter release from the photoreceptor axons and interneurons, refining their representations of visual information in changing conditions.

\section{The dSK fly model}

To our knowledge, this study provides the first description of an SK animal genetic model. Unlike mammals and C. elegans, which have three and four SK genes, respectively (Köhler et al., 1996; Salkoff et al., 2005), the fruit fly genome contains a single highly conserved $S K$ gene $(d S K)$ that encodes a remarkably similar current to the mammalian counterpart. Our report is also the first to evaluate the impact of SK channel on neuronal and network functions, which affect adaptation and signaling performance of photoreceptors. Because of this new model system, and the new insight it has given about sophisticated network functions, it is now possible to start dissecting the contributions of dSK channel and dSK-expressing cells in circuits, involved in complex behaviors, addiction, and learning and memory.

\section{Homeostatic sensitivity regulation}

The photoreceptor output is sign-inverted by LMCs/ACs' histamine-receptors and then partially fed back to photoreceptors through synaptic conductances (Fig. 10). Darkening hyperpolarizes photoreceptors, reducing their tonic histamine release (Uusitalo et al., 1995b; Zheng et al., 2006). This in turn depolarizes LMCs/ACs, increasing their feedbacks to photoreceptor axons (Zheng et al., 2006). These excitatory conductances can explain why in the dark, photoreceptors of the fully functioning network are more depolarized than the dissociated photoreceptors, which lack axons.

At the photoreceptor terminals, the slowly hyperpolarizing $\mathrm{K}^{+}$conductances of dSK channels (Fig. $2 \mathrm{~B}$ ) are likely to facilitate local inhibition by offsetting voltage-dependent $\mathrm{Ca}^{2+}$ increases, as a part of axonal sensitivity control that refines waveforms and patterning of presynaptic signals (Fig. 10 A). Here, dSK might be also required in fine-tuning histamine release, whereby any synaptic transmission defect would lead to increased feedback inputs into photoreceptor axons (Fig. 10B). These scenarios (Fig. $10 A, B)$ are not mutually exclusive and in the absence of dSK would result in increased synaptic feedback conductances from the lamina interneurons, leading to the observed lowered input resistance and the more depolarized resting potential of photoreceptors. They are further supported by our expression data, and the intact morphology and phototransduction machinery in $d S K^{-}$photoreceptors.

During and following prolonged light exposure, both $d S K^{-}$photoreceptor and LMC voltage responses oscillated in an activitydependent manner, implying that in wild-type, dSK channels would have a direct role as adaptive dampers in synaptic communication. It is possible that these perturbations resulted from faulty synaptic gain control in dSK-expressing nonglutamatergic interneurons, because inactivation of dSK only in photoreceptors was not sufficient to induce them, and because in mutant, dSK seemed not expressed in glutamatergic interneurons (ACs and L2s), which synapse directly to photoreceptor axons. Furthermore, photoreceptor oscillations were strengthened after light adaptation and to dim but not bright luminances. Such stimulus conditions lower presynaptic (photoreceptor) potentials and, therefore, are expected to boost postsynaptic feedbacks from the network. Thus, in dim luminances, mistuned synaptic feedbacks of high gain could transfer energy to wrong stimulus frequencies, oscillating the photoreceptor output. Collectively, these findings hint that oscillations might originate from $d S K^{-} \mathrm{L} 4$ monopolar cell synapses, 

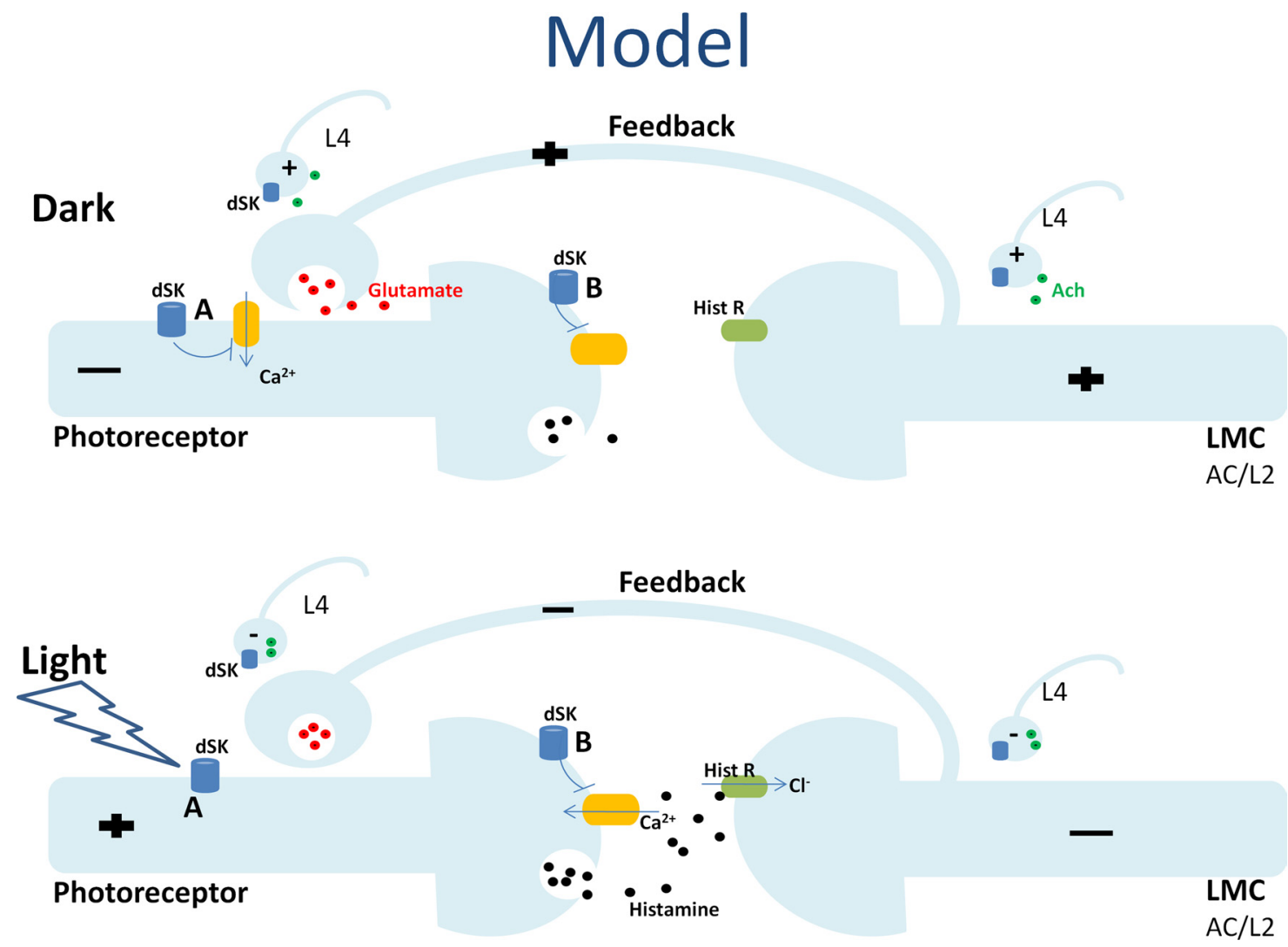

Figure 10. dSK contributes to photoreceptor performance by fine-tuning synaptic transmission at the photoreceptor-LMC-photoreceptor network. In this model, dSK is expressed in photoreceptor axons, while glutamatergic AC interneurons and L2 monopolar cells, which form the direct synaptic feedbacks to photoreceptor axons, do not express dSK. In addition, photoreceptor axons and $A C / L 2$ receive extra inputs from the lamina network, through functional contacts with nonglutamatergic LMCs that express dSK, including L4s. Within R1-R6 axons, dSK counteracts Ca ${ }^{2+}$ and fine-tunes neurotransmitter release. In the dark, LMCs receive less feedforward input from photoreceptors and are, therefore, more depolarized. In the absence of dSK, the observed depolarized resting potential and the lowered input resistance can be attributed to an inability to zero photoreceptor axonal voltages $(\boldsymbol{A})$ and/or increased feedback synaptic inputs into photoreceptor axons due to misregulated histamine release $(\boldsymbol{B})$. In the light, photoreceptors are more depolarized and, therefore, the feedback input is reduced (both direct and extra). In the dark/dim conditions, an inability to fine-tune neurotransmitter release from nonglutamatergic LMCs (most likely L4), at least partially, can lead to the observed oscillatory responses in both photoreceptors and LMCs (particularly in L2s) in $d S K^{-}$flies.

which feedback to both photoreceptor axons and L2 monopolar cells (Fig. 10) in the same and neighboring lamina cartridges (Meinertzhagen and O'Neil, 1991), and thus are ideally placed to mediate adaptive network functions (Strausfeld and CamposOrtega, 1977).

To maximize visual information, network adaptation to dim environment involves integration over space and time, whereupon functional connectivity increases between cells (redundancy), smoothing low signal-to-noise images. But when adapting to bright environment its cells operate more independently, as lateral and temporal inhibition reduces redundancies to sharpen high signal-tonoise images (van Hateren, 1992). Thus, we speculate that faulty gain control in the lamina branches of $d S K^{-} \mathrm{L} 4$ monopolar cells would hinder such connectivity transitions between dark- and lightadapted network states; affecting the rate of adaptation in photoreceptor and LMC outputs and making them more susceptible to oscillations. This view is further supported by the recording statistics from $d S K^{-}$flies: all R1-R6 receive inputs from L4, and correspondingly most photoreceptor outputs showed suboptimal adaptation and oscillated; only $\mathrm{L} 2$ monopolar cells receive inputs from L 4 and only $\sim 40 \%$ of LMC outputs oscillated. These observations highlight how the neuronal functions in the early motion pathways can depend upon adaptive gain control, leading to different behavioral outcomes in different stimulus conditions (Zheng et al., 2006; Rister et al., 2007; Katsov and Clandinin, 2008; Nikolaev et al., 2009; Zhu et al., 2009; Joesch et al., 2010).

\section{Robustness of dynamic coding in $d S K^{-}$mutant photoreceptors}

Despite the fast oscillatory responses, $d S K^{-}$photoreceptors revealed a near-normal encoding capacity. The decreased input resistance in $d S K^{-}$photoreceptors is similar to that found in Shaker and Shab mutant photoreceptors (Niven et al., 2003; Vähäsöyrinki et al., 2006), where it has been argued to compensate for mutant defects and underlie the robustness of encoding. In Shaker photoreceptors, the decrease in input resistance partially restores the efficient use of the operating voltage range (Niven et al., 2003). Conversely, $d S K^{-}$photoreceptors, like Shab, show remarkable robustness in their light-voltage relationships, sensitivities, and reliability of dynamic encoding (rate of information transfer).

Although it is unclear whether the underlying mechanisms are the same, the lower input resistances in all these mutant photoreceptors are believed to be direct manifestations of compensation. In this study, the lowered input resistance, measured from intact $d S K^{-}$photoreceptors in vivo, indicates an increase in conductance at the photoreceptor axon. Here we propose that this compensation results from feedback synaptic inputs from the neighboring interneurons, because everything else being equal, instead of depolarizing $d S K^{-}$photoreceptors, excess of $\mathrm{K}^{+}$ and/or $\mathrm{Cl}^{-}$leak-conductances would work to hyperpolarize the cells toward the reverse potentials of these ions $(-80 \mathrm{mV}$ ) (Niven et al., 2003). Thus, even if such leaks existed-a possibility that we 
cannot exclude - they would be masked by the depolarizing synaptic conductances from the network. Furthermore, response dynamics of photoreceptors, with inactivated dSK channels, were closer to wild-type when the network was normal rather than mutated (cf. Fig. 9A), implying that extrinsic conductances (from the network) shape photoreceptor output more than intrinsic leak conductances (which, if dominating, should produce identical outputs for the two cases).

The faster kinetics and retuned adapting properties of $d S K^{-}$ photoreceptors impose a constant high energy cost to maintain both a low input resistance and a depolarized resting potential in the dark, suggesting that the mutants are at a clear disadvantage. Thus, managing energy costs is a powerful evolutionary objective (de Polavieja, 2002), which together with noise and various behavioral objectives (de Polavieja, 2004), supposedly refined the molecular constituents of the lamina network to overcome the limitations of its unreliable, slow hardware.

\section{References}

Barlow H (1961) Possible principles underlying the transformations of sensory messages. In: Sensory communication, Chap 13 (WA Rosenblith, ed), pp 217-234. Cambridge, MA: MIT.

Blatz AL, Magleby KL (1986) Single apamin-blocked Ca-activated K+ channels of small conductance in cultured rat skeletal muscle. Nature 323:718-720.

Chinchore Y, Mitra A, Dolph PJ (2009) Accumulation of rhodopsin in late endosomes triggers photoreceptor cell degeneration. PLoS Genet 5: e1000377.

Clark BD, Kurth-Nelson ZL, Newman EA (2009) Adenosine-evoked hyperpolarization of retinal ganglion cells is mediated by G-protein-coupled inwardly rectifying $\mathrm{K}^{+}$and small conductance $\mathrm{Ca}^{2+}$-activated $\mathrm{K}^{+}$channel activation. J Neurosci 29:11237-11245.

de Polavieja GG (2002) Errors drive the evolution of biological signalling to costly codes. J Theor Biol 214:657-664.

de Polavieja GG (2004) Reliable biological communication with realistic constraints. Phys Rev E Stat Nonlin Soft Matter Phys 70:061910.

Faber ES, Delaney AJ, Sah P (2005) SK channels regulate excitatory synaptic transmission and plasticity in the lateral amygdala. Nat Neurosci 8:635-641.

Fischbach KF, Dittrich AP (1989) The optic lobe of Drosophila melanogaster. I. A Golgi analysis of wild-type structure. Cell Tissue Res 258:441-475.

Gengs C, Leung HT, Skingsley DR, Iovchev MI, Yin Z, Semenov EP, Burg MG, Hardie RC, Pak WL (2002) The target of Drosophila photoreceptor synaptic transmission is a histamine-gated chloride channel encoded by ort (hclA). J Biol Chem 277:42113-42120.

Gho M, Mallart A (1986) Two distinct calcium-activated potassium currents in larval muscle fibres of Drosophila melanogaster. Pflugers Arch 407:526-533.

Gonzalez-Bellido PT, Wardill TJ, Juusola M (2011) Compound eyes and retinal information processing in miniature dipteran species match their specific ecological demands. Proc Natl Acad Sci U S A 108:4224-4229.

Gu Y, Oberwinkler J, Postma M, Hardie RC (2005) Mechanisms of light adaptation in Drosophila photoreceptors. Curr Biol 15:1228-1234.

Hallworth NE, Wilson CJ, Bevan MD (2003) Apamin-sensitive small conductance calcium-activated potassium channels, through their selective coupling to voltage-gated calcium channels, are critical determinants of the precision, pace, and pattern of action potential generation in rat subthalamic nucleus neurons in vitro. J Neurosci 23:7525-7542.

Hardie RC (1987) Is histamine a neurotransmitter in insect photoreceptors? J Comp Physiol A 161:201-213.

Hardie RC (1989) A histamine-activated chloride channel involved in neurotransmission at a photoreceptor synapse. Nature 339:704-706.

Hardie RC (1995) Effects of intracellular $\mathrm{Ca}^{2+}$ chelation on the light response in Drosophila photoreceptors. J Comp Physiol A 177:707-721.

Hardie RC, Raghu P (2001) Visual transduction in Drosophila. Nature 413:186-193.

Hardie RC, Voss D, Pongs O, Laughlin SB (1991) Novel potassium channels encoded by the Shaker locus in Drosophila photoreceptors. Neuron 6:477-486.
Hardie RC, Martin F, Cochrane GW, Juusola M, Georgiev P, Raghu P (2002) Molecular basis of amplification in Drosophila phototransduction: roles for G protein, phospholipase C, and diacylglycerol kinase. Neuron 36:689-701.

Ishii TM, Maylie J, Adelman JP (1997) Determinants of apamin and d-tubocurarine block in SK potassium channels. J Biol Chem 272: 23195-23200.

Joesch M, Schnell B, Raghu SV, Reiff DF, Borst A (2010) ON and OFF pathways in Drosophila motion vision. Nature 468:300-304.

Juusola M, de Polavieja GG (2003) The rate of information transfer of naturalistic stimulation by graded potentials. J Gen Physiol 122:191-206.

Juusola M, Hardie RC (2001) Light adaptation in Drosophila photoreceptors: I. Response dynamics and signaling efficiency at 25 degrees C. J Gen Physiol 117:3-25.

Juusola M, Kouvalainen E, Järvilehto M, Weckström M (1994) Contrast gain, signal-to-noise ratio, and linearity in light-adapted blowfly photoreceptors. J Gen Physiol 104:593-621.

Juusola M, Uusitalo RO, Weckström M (1995) Transfer of graded potentials at the photoreceptor-interneuron synapse. J Gen Physiol 105:117148.

Katsov AY, Clandinin TR (2008) Motion processing streams in Drosophila are behaviorally specialized. Neuron 59:322-335.

Klöcker N, Oliver D, Ruppersberg JP, Knaus HG, Fakler B (2001) Developmental expression of the small-conductance $\mathrm{Ca}(2+)$-activated potassium channel SK2 in the rat retina. Mol Cell Neurosci 17:514-520.

Köhler M, Hirschberg B, Bond CT, Kinzie JM, Marrion NV, Maylie J, Adelman JP (1996) Small-conductance, calcium-activated potassium channels from mammalian brain. Science 273:1709-1714.

Kolodziejczyk A, Sun X, Meinertzhagen IA, Nässel DR (2008) Glutamate, GABA and acetylcholine signaling components in the lamina of the Drosophila visual system. PLoS One 3:e2110.

Komatsu A, Singh S, Rathe P, Wu CF (1990) Mutational and gene dosage analysis of calcium-activated potassium channels in Drosophila: correlation of micro- and macroscopic currents. Neuron 4:313-321.

Kwon Y, Montell C (2006) Dependence on the Lazaro phosphatidic acid phosphatase for the maximum light response. Curr Biol 16:723-729.

Laughlin SB, de Ruyter van Steveninck RR, Anderson JC (1998) The metabolic cost of neural information. Nat Neurosci 1:36-41.

Mahr A, Aberle H (2006) The expression pattern of the Drosophila vesicular glutamate transporter: a marker protein for motoneurons and glutamatergic centers in the brain. Gene Expr Patterns 6:299-309.

Meinertzhagen IA, O'Neil SD (1991) Synaptic organization of columnar elements in the lamina of the wild type in Drosophila melanogaster. J Comp Neurol 305:232-263.

Meinertzhagen IA, Sorra KE (2001) Synaptic organization in the fly's optic lamina: few cells, many synapses and divergent microcircuits. Prog Brain Res 131:53-69.

Ngo-Anh TJ, Bloodgood BL, Lin M, Sabatini BL, Maylie J, Adelman JP (2005) SK channels and NMDA receptors form a $\mathrm{Ca}^{2+}$-mediated feedback loop in dendritic spines. Nat Neurosci 8:642-649.

Nikolaev A, Zheng L, Wardill TJ, O’Kane CJ, de Polavieja GG, Juusola M (2009) Network adaptation improves temporal representation of naturalistic stimuli in Drosophila eye: II mechanisms. PLoS One 4:e4306.

Niven JE, Vähäsöyrinki M, Kauranen M, Hardie RC, Juusola M, Weckström M (2003) The contribution of Shaker K+ channels to the information capacity of Drosophila photoreceptors. Nature 421:630-634.

Nolting A, Ferraro T, D’hoedt D, Stocker M (2007) An amino acid outside the pore region influences apamin sensitivity in small conductance $\mathrm{Ca}^{2+}$. activated K+ channels. J Biol Chem 282:3478-3486.

Parks AL, Cook KR, Belvin M, Dompe NA, Fawcett R, Huppert K, Tan LR, Winter CG, Bogart KP, Deal JE, Deal-Herr ME, Grant D, Marcinko M, Miyazaki WY, Robertson S, Shaw KJ, Tabios M, Vysotskaia V, Zhao L, Andrade RS, et al. (2004) Systematic generation of high-resolution deletion coverage of the Drosophila melanogaster genome. Nat Genet 36:288-292.

Pedarzani P, Mosbacher J, Rivard A, Cingolani LA, Oliver D, Stocker M, Adelman JP, Fakler B (2001) Control of electrical activity in central neurons by modulating the gating of small conductance $\mathrm{Ca}^{2+}$-activated $\mathrm{K}+$ channels. J Biol Chem 276:9762-9769.

Raghu SV, Borst A (2011) Candidate glutamatergic neurons in the visual system of Drosophila. PLoS One 5:e19472.

Reinke R, Krantz DE, Yen D, Zipursky SL (1988) Chaoptin, a cell surface 
glycoprotein required for Drosophila photoreceptor cell morphogenesis, contains a repeat motif found in yeast and human. Cell 52:291-301.

Rister J, Pauls D, Schnell B, Ting CY, Lee CH, Sinakevitch I, Morante J, Strausfeld NJ, Ito K, Heisenberg M (2007) Dissection of the peripheral motion channel in the visual system of Drosophila melanogaster. Neuron 56:155-170.

Salkoff L, Wei AD, Baban B, Butler A, Fawcett G, Ferreira G, Santi CM (2005) Potassium channels in C. elegans. WormBook 30:1-15.

Sanes JR, Zipursky SL (2010) Design principles of insect and vertebrate visual systems. Neuron 66:15-36.

Shannon CE (1948) A mathematical theory of communication. Bell Syst Tech J 27:379-423.

Shatz CJ (1990) Impulse activity and the patterning of connections during CNS development. Neuron 5:745-756.

Shaw SR (1984) Early visual processing in insects. J Exp Biol 112:225-251.

Sinakevitch I, Strausfeld NJ (2004) Chemical neuroanatomy of the fly's movement detection pathway. J Comp Neurol 468:6-23.

Singh S, Wu CF (1989) Complete separation of four potassium currents in Drosophila. Neuron 2:1325-1329.

Stocker M (2004) Ca(2+)-activated K+ channels: molecular determinants and function of the SK family. Nat Rev Neurosci 5:758-770.

Strausfeld NJ, Campos-Ortega JA (1977) Vision in insects: pathways possibly underlying neural adaptation and lateral inhibition. Science 195: 894-897.

Uusitalo RO, Juusola M, Weckström M (1995a) Graded responses and spiking properties of identified first-order visual interneurons of the fly compound eye. J Neurophysiol 73:1782-1792.

Uusitalo RO, Juusola M, Kouvalainen E, Weckström M (1995b) Tonic transmitter release in a graded potential synapse. J Neurophysiol 74:470-473.

Vähäsöyrinki M, Niven JE, Hardie RC, Weckström M, Juusola M (2006) Robustness of neural coding in Drosophila photoreceptors in the absence of slow delayed rectifier $\mathrm{K}^{+}$channels. J Neurosci 26:2652-2660.

van Hateren JH (1992) A theory of maximizing sensory information. Biol Cybern 68:23-29. van Hateren JH (1997) Processing of natural time series of intensities by the visual system of the blowfly. Vision Res 37:3407-3416.

Van Vactor D Jr, Krantz DE, Reinke R, Zipursky SL (1988) Analysis of mutants in chaoptin, a photoreceptor cell-specific glycoprotein in Drosophila, reveals its role in cellular morphogenesis. Cell 52:281-290.

Wagh DA, Rasse TM, Asan E, Hofbauer A, Schwenkert I, Dürrbeck H, Buchner S, Dabauvalle MC, Schmidt M, Qin G, Wichmann C, Kittel R, Sigrist SJ, Buchner E (2006) Bruchpilot, a protein with homology to ELKS/ CAST, is required for structural integrity and function of synaptic active zones in Drosophila. Neuron 49:833-844.

Wang GY, Olshausen BA, Chalupa LM (1999) Differential effects of apamin- and charybdotoxin-sensitive $\mathrm{K}^{+}$conductances on spontaneous discharge patterns of developing retinal ganglion cells. J Neurosci 19: 2609-2618.

Wang T, Xu H, Oberwinkler J, Gu Y, Hardie RC, Montell C (2005) Light activation, adaptation, and cell survival functions of the $\mathrm{Na}^{+} / \mathrm{Ca}^{2+} \mathrm{ex}-$ changer CalX. Neuron 45:367-378.

Weckström M, Laughlin SB (1995) Visual ecology and voltage-gated ion channels in insect photoreceptors. Trends Neurosci 18:17-21.

Wolfart J, Neuhoff H, Franz O, Roeper J (2001) Differential expression of the small-conductance, calcium-activated potassium channel SK3 is critical for pacemaker control in dopaminergic midbrain neurons. J Neurosci 21:3443-3456.

Xia XM, Fakler B, Rivard A, Wayman G, Johnson-Pais T, Keen JE, Ishii T, Hirschberg B, Bond CT, Lutsenko S, Maylie J, Adelman JP (1998) Mechanism of calcium gating in small-conductance calcium-activated potassium channels. Nature 395:503-507.

Zheng L, de Polavieja GG, Wolfram V, Asyali MH, Hardie RC, Juusola M (2006) Feedback network controls photoreceptor output at the layer of first visual synapses in Drosophila. J Gen Physiol 127:495-510.

Zheng L, Nikolaev A, Wardill TJ, O'Kane CJ, de Polavieja GG, Juusola M (2009) Network adaptation improves temporal representation of naturalistic stimuli in Drosophila eye: I dynamics. PLoS One 4:e4307.

Zhu Y, Nern A, Zipursky SL, Frye MA (2009) Peripheral visual circuits functionally segregate motion and phototaxis behaviors in the fly. Curr Biol 19:613-619. 